\title{
NEW BOUND-STATE SOLUTIONS OF THE DEFORMED KLEIN-GORDON AND SCHRÖDINGER EQUATIONS FOR ARBITRARY L-STATE WITH THE MODIFIED EQUAL VECTOR AND SCALAR MANNING-ROSEN PLUS A CLASS OF YUKAWA POTENTIALS IN RNCQM AND NRNCQM SYMMETRIES
}

\author{
Abdelmadjid Maireche (iD) \\ Laboratory of Physics and Material Chemistry, Physics department, \\ Sciences Faculty, University of M'sila \\ BP 239 Chebilia-M'sila, Algeria \\ abdelmadjid.maireche@univ-msila.dz
}

(Received 30 June 2021; in final form 14 July 2021; accepted 19 August 2021; published online 19 November 2021)

\begin{abstract}
In this work, we employed the elegant tool of Bopp's shift and standard perturbation theory methods to obtain a new relativistic and nonrelativistic approximate bound state solution of the deformed Klein-Gordon and deformed Schrödinger equations using the modified equal vector scalar Manning-Rosen plus a class of Yukawa potentials (DVSMCY-Ps, in short) model. Furthermore, we have employed the improved approximation to the centrifugal term for some selected diatomic molecules, such as $\mathrm{N}_{2}, \mathrm{I}_{2}, \mathrm{HCl}, \mathrm{CH}, \mathrm{LiH}$, and $\mathrm{CO}$, in the symmetries of extended quantum mechanics to obtain the approximate solutions. The relativistic shift energy $\Delta E_{\text {mcy }}^{\text {tot }}\left(n, \delta, \eta, b, A, V_{0}, V_{0}^{\prime}, \Theta, \sigma, \chi, j, l, s, m\right)$ and the perturbative nonrelativistic corrections $\Delta E_{\mathrm{mcy}}^{\mathrm{nr}}\left(n, \delta, \eta, b, A, V_{0}, V_{0}^{\prime}, \Theta, \sigma, \chi, j, l, s, m\right)$ appeared as a function of the parameters $\left(\delta, \eta, b, A, V_{0}, V_{0}^{\prime}\right)$, the parameters of noncommutativity $(\Theta, \sigma, \chi)$, in addition to the atomic quantum numbers $(n, j, l, s, m)$. In both relativistic and nonrelativistic problems, we show that the corrections to the spectrum energy are smaller than the main energy in the ordinary cases of relativistic quantum mechanics and nonrelativistic quantum mechanics. A straightforward limit of our results to ordinary quantum mechanics shows that the present result under DVSMCY-Ps is consistent with what is obtained in the literature. In the new symmetries of noncommutative quantum mechanics, it is not possible to get exact analytical solutions for $l=0$, and $l \neq 0$ can only be solved approximately. We have observed that the DKGE under the DVSMCY-Ps model has a physical behavior similar to the Duffin-Kemmer equation for meson with spin-1, it can describe a dynamic state of a particle with spin-1 in the symmetries of relativistic noncommutative quantume mechanics.

Key words: Klein-Gordon equation, Schrödinger equation, Manning-Rosen potential, class of Yukawa potentials, the diatomic molecules, noncommutative geometry, Bopp's shift method and star products.
\end{abstract}

DOI: https://doi.org/10.30970/jps.25.4301

\section{INTRODUCTION}

During the last decades, the research arena has witnessed great progress of outstanding scientific value in various fields of physics and chemistry where many exact and approximate solutions have been achieved for the three fundamental equations in both nonrelativistic and relativistic regimes. This is done in the domains of low or high energies at microscopic and subatomic scales by using the most popular methods such as the Nikiforov-Uvarov method [1], the Wentzel-KramersBrillouin method [2], the proper quantization rule [3], the exact quantization rule [4] and in addition to many other methods. The exact solutions of the fundamental equations are only possible in some exceptional cases like the Harmonic oscillator and the Hydrogen atom as a typical model. As for most of the cases considered by researchers, it is done by approximate and numerical methods such as the Pekeris approximation [5], the Greene and Aldrich approximation [6], the good approximation proposed by Yazarloo et al. in the study of the oscillator strengths based on the Möbius square potential under the Schrödinger equation [7, 8]. Currently, physicists in general and theorists in particular have developed much interest in searching for exponential-type potentials. The predominant reason for this is that this type of potential has many different and important applications, for example, the Manning-Rosen potential can be applied to various fields such as atomic, condensed matter, particle, and nuclear physics in both relativistic and nonrelativistic regimes [9-12]. Furthermore, it is used to describe the vibrations of diatomic molecules such as $\mathrm{N}_{2}$, $\mathrm{I}_{2}, \mathrm{HCl}, \mathrm{CH}, \mathrm{LiH}$, and $\mathrm{CO}$ [13]. Many authors have studied Manning-Rosen potential in the nonrelativistic case, in both the $s$ and $l$-waves cases (see, for example, [1415]). On the other hand, this potential was also studied in the relativistic regimes of the Klein-Gordon and Dirac equations [16-19].

The Yukawa potentials are another type of exponential potentials [20] (also known as static screened Coulomb potentials) that have received a great deal of attention, in many fields of physics such as nuclear physics, atomic physics, solid-state physics, and astrophysics and they were studied in both relativistic and nonrelativistic quantum mechanics. They areused to descri- 


\section{A. MAIRECHE}

be the interactions of hydrogen-like atoms (such as $\mathrm{He}^{+}$ , $\mathrm{Li}^{+2}$, and $\mathrm{Be}^{+}$) and neutral atoms ( such as ${ }^{22} \mathrm{Na},{ }^{12} \mathrm{C}$, and ${ }^{158} \mathrm{Au}$ ) [21-29].

Recently, there has been great interest in combining two or more potentials to have a large range of applications, for example, the Manning-Rosen plus a class of Yukawa potentials. In 2017, B. I. Ita et al. [30] studied the Dirac equation for the Manning-Rosen plus a class of Yukawa potentials and Coulomb-like tensor interaction. In the next year, H. Louis et al. [31] analyzed the Klein-Gordon equation (KGE) under the same potential but with both Pekeris-like approximation of the Coulomb term and parametric Nikiforov-Uvarov method. Very recently, A. I. Ahmadov et al. [32] studied the Klein-Gordon equation under the linear combination of Manning-Rosen and a class of Yukawa potentials using both the Nikiforov-Uvarov, SUSYQM methods and the approximation scheme proposed by Greene and Aldrich, and obtained the relativistic energy spectrum for any $l$-state and the corresponding radial wave functions. This new combination is useful in studying the nucleus deformed-pair interactions and spin-orbit coupling.

As a result of several considerations and many physical problems apparat at the level of the non-renormalizable electroweak interaction, the nonregularization of quantum field theories, quantum gravity, string theory, where the idea of non-commutativity resulting from properties of the deformation of spacespace (W. Heisenberg in 1930 was the first to suggest the idea and then it was developed by H. Snyder in 1947) was one of the major solutions to these problems. Researchers have received great praise for this approach through some related work [33-44]. Naturally, the topographical properties of the noncommutativity space-space and phase-phase have a clear effect on the various physical properties of quantum systems and this has been a very interesting subject in many fields of physics as mentioned previously.

In the last few years, we have investigated many interesting studies concerned with the Yukawa potential in a nonrelativistic and relativistic state [45-49] due to the importance of its applications in many fields, as previously indicated. From what we have seen so far, most of the studies concerning Manning-Rosen and the class of Yukawa potentials were within the framework of ordinary quantum mechanics.

The above works motivated us to investigate the approximate solutions of the 3-dimensional deformed Klein-Gordon equation (DKGE) and deformed Schrödinger equation (DSE) for the modified equal vector scalar Manning-Rosen plus a class of Yukawa potentials (DVSMCY-Ps) model offered by A. I. Ahmadov and his coworkers [32] in relativistic quantum mechanics (RQM). The potential under study can be applied to some selected diatomic molecules such as $(\mathrm{HCl}, \mathrm{CH}, \mathrm{LiH}$, and $\mathrm{CO})$ in relativistic noncommutative quantume mechanics (RNCQM) and nonrelativistic noncommutative quantume mechanics (NRNCQM) symmetries. Upon concuction more studies in the microscopic scales, we hope to gain more scientific knowledge of elementary particles in the field of nano-scales. The relativistic and nonrelativistic energy levels under the DVSMCY-Ps have not been obtained yet in the RNCQM and NRNCQM symmetries. We hope to find new applications and profound physical interpretations using a new version model of the DVSMCY-Ps, this potential modeled in the new symmetries of noncommutative quantume mechanics (NCQM) as follows:

$$
\begin{aligned}
V_{\mathrm{mcy}}(r) & =\frac{1}{2 M b^{2}}\left[\frac{\eta(\eta-1) e^{-2 r / b}}{\left(1-e^{-r / b}\right)^{2}}-\frac{A e^{-r / b}}{1-e^{-r / b}}\right]-\frac{V_{0} e^{-\delta r}}{r}-\frac{V_{0}^{\prime} e^{-2 \delta r}}{r^{2}} \mapsto V_{\mathrm{mcy}}\left(r_{\mathrm{nc}}\right) \\
& =V_{\mathrm{mcy}}(r)-\frac{\partial V_{\mathrm{msy}}(r)}{\partial r} \frac{\mathbf{L} \Theta}{2 r}+O\left(\Theta^{2}\right), \\
S_{\mathrm{mcy}}(r) & =\frac{1}{2 M b^{2}}\left[\frac{\lambda(\lambda-1) e^{-2 r / b}}{\left(1-e^{-r / b}\right)^{2}}-\frac{B e^{-r / b}}{1-e^{-r / b}}\right]-\frac{S_{0} e^{-\delta r}}{r}-\frac{S_{0}^{\prime} e^{-2 \delta r}}{r^{2}} \mapsto S_{\mathrm{mcy}}\left(r_{\mathrm{nc}}\right) \\
& =S_{\mathrm{mcy}}(r)-\frac{\partial S_{\mathrm{msy}}(r)}{\partial r} \frac{\mathbf{L} \Theta}{2 r}+O\left(\Theta^{2}\right) .
\end{aligned}
$$

Here, $V_{\text {mcy }}(r)$ and $S_{\text {mcy }}(r)$ are vector and scalar Manning-Rosen plus a class of Yukawa potentials in the symmetries of ordinary quantum mechanics are given by $[32], \vec{L} \vec{\Theta}=\mathbf{L} \Theta$, the parameter $b$ relates to the potential range, while $A(B)$ and $\eta(\lambda)$ are four dimensionless parameters, $\left(V_{0}, V_{0}^{\prime}\right)$ and $\left(S_{0}, S_{0}^{\prime}\right)$ are the strengths of the class Yukawa potential, and its range is $1 / \delta ; r_{\mathrm{nc}}$ and $r$ are the distances between the two particles in NCQM and QM symmetries. The coupling $\mathbf{L} \Theta$ equals $L_{x} \Theta_{12}+L_{y}$ $\Theta_{23}+L_{z} \Theta_{13}$ with $L_{x}, L_{y}$ and $L_{z}$ representing the usual components of the angular momentum operator $\mathbf{L}$ in RQM and NRQM, while the new noncommutativity 
parameter $\Theta_{i j}$ equals $\theta_{i j} / 2$. The new algebraic structure of covariant noncommutative canonical commutations relations (NCNCCRs) in the three representations of the Schrödinger, Heisenberg, and interactions pictures, in the new symmetry of NCQM, is as follows [50-60]:

$$
\begin{aligned}
& {\left[\hat{x}_{\mu}^{\mathrm{S} *}, \hat{p}_{\nu}^{\mathrm{S}}\right]=\left[\hat{x}_{\mu}^{\mathrm{H} *}, \hat{p}_{\nu}^{\mathrm{H}}\right]=\left[\hat{x}_{\mu}^{\mathrm{I}}, \hat{p}_{\nu}^{\mathrm{I}}\right]=i \hbar_{\mathrm{eff}} \delta_{\mu \nu},} \\
& {\left[\hat{x}_{\mu}^{\mathrm{S} *}, \hat{x}_{\nu}^{\mathrm{S}}\right]=\left[\hat{x}_{\mu}^{\mathrm{H} *}, \hat{x}_{\nu}^{\mathrm{H}}\right]=\left[\hat{x}_{\mu}^{\mathrm{I}}{ }^{*} \hat{x}_{\nu}^{\mathrm{I}}\right]=i \theta_{\mu \nu} .}
\end{aligned}
$$

It is important to note that Eq. (1.4) is a covariant equation (the same behavior of $x^{\mu}$ ) under the Lorentz transformation, which includes boosts and/or rotations of the observer's inertial frame. We generalize the NCNCCRs to include the Heisenberg and interaction pictures. It should be noted that, in our calculation, we have used the natural units $\hbar=c=1$. Here, $\hbar_{\text {eff }} \cong \hbar$ is the effective Planck constant, $\theta_{\mu \nu}=\epsilon_{\mu \nu} \theta$ ( $\theta$ is the noncommutative parameter with the dimension of [length $]^{2}$ which present the fluidity of the space, and $\epsilon_{\mu \nu}$ is just an antisymmetric number, for example $\epsilon_{12}=-\epsilon_{21}=1$, $\left.\epsilon_{13}=-\epsilon_{31}=1, \epsilon_{23}=-\epsilon_{32}=1, \epsilon_{11}=\epsilon_{22}=\epsilon_{33}=0\right)$, which is an infinitesimal parameter if compared to the energy values and elements of antisymmetric $3 \times 3$ real matrices, and $\delta_{\mu \nu}$ is the Kronecker symbol. The symbol $*$ denotes the Weyl-Moyal star product, which is generalized between two ordinary functions $f(x) h(x)$ to the new deformed form $\hat{f}(\hat{x}) \hat{h}(\hat{x})$ being expressed with the WeylMoyal star product $f(x) * h(x)$ in the symmetries of NCQM as follows [57-69]:

$$
\left.(f * h)(x)=\exp \left(i \epsilon^{\mu \nu} \theta \partial_{\mu}^{x} \partial_{\nu}^{x}\right)(f h)(x) \approx(f h)(x)-\frac{i \epsilon^{\mu \nu} \theta}{2} \partial_{\mu}^{x} f \partial_{\mu}^{x} h\right\rfloor_{x^{\mu}=x^{\nu}}+O\left(\theta^{2}\right)
$$

The indices $\mu, \nu=1,2,3$ and $O\left(\theta^{2}\right)$ stands for the second and higher-order terms of the NC parameter. On the other hand, the choice of these combinations of the Manning-Rosen plus a class of Yukawa potentials stems from the fact that it exhibits an almost exact behavior similar to the Morse [70] and Deng-Fan-Eckart [71] potentials and so we considered it to be an excellent choice for the study of the atomic interaction for diatomic molecules such as $\mathrm{HCl}, \mathrm{CH}, \mathrm{LiH}$, and $\mathrm{CO}$. Our current work is structured in six sections. The first one includes the scope and purpose of our investigation while the remaining parts of the paper are structured as follows. A review of the KGE with the equal vector scalar ManningRosen plus a class of Yukawa potentials is presented in Section II. Section III is devoted to studying the DRKGE by applying the ordinary Bopp's shift method and improved approximation of the centrifugal term to obtain the effective potential of DVSMCY-Ps. Besides, via perturbation theory we find the expectation values of some radial terms to calculate the energy shift produced with the effect of the perturbed effective potential of DVSMCY-Ps. Section IV is devoted to present the global energy shift and the global energy spectra produced with DVSMCY-Ps in the RNCQM symmetries. In Section $\mathrm{V}$, we determine the energy spectra of some selected diatomic molecules such as $(\mathrm{HCl}, \mathrm{CH}, \mathrm{LiH}$, and $\mathrm{CO}$ ) under DVSMCY-Ps in the RNCQM. In Section VI, the summary and conclusion are presented.

\section{REVISED RKGE UNDER THE EQUAL VECTOR SCALAR MANNING-ROSEN PLUS A CLASS OF YUKAWA POTENTIALS MODEL IN RQM}

The 3-dimensional relativistic Klein-Gordon equation (RKGE) with a scalar potential $S_{\text {mcy }}(r)$ and a vector potential $V_{\text {mcy }}(r)$ for the diatomic molecule with reduced mass $M$ and wave function is given as

$$
\left(\begin{array}{c}
-\Delta+\left(M+S_{\mathrm{mcy}}(r)\right)^{2} \\
-\left(E_{n l}-V_{\mathrm{mcy}}(r)\right)^{2}
\end{array}\right) \Psi(r, \theta, \varphi)=0 .
$$

The vector potential $V_{\text {mcy }}(r)$ is due to the four-vector linear momentum operator $A^{\mu}\left(V_{\text {mcy }}(r), \mathbf{A}=\mathbf{0}\right)$ and the space-time scalar potential $S_{\mathrm{mcy}}(r)$ is due to the effect of the mass $M ; E_{n l}$ represents the relativistic energy eigenvalues in 3-dimensions and $l$ represents the principal and orbital quantum numbers, respectively. The Manning-Rosen plus a class of Yukawa potentials model has spherical symmetry, allowing the solutions of the time-independent RKGE of the known form $\Psi(r, \theta, \varphi)=$ $\frac{U_{n l}(r)}{r} Y_{l}^{m}(\theta, \varphi)$ to separate the radial $U_{n l}(r)$ and angular parts $Y_{l}^{m}(\theta, \varphi)$ of the wave function $\Psi(r, \theta, \varphi)$ and $\Delta$ is the ordinary 3-dimensional Laplacian operator. Thus, Eq. (2.1) becomes:

$$
\left(\begin{array}{c}
\frac{d^{2}}{d r^{2}}-\left(M^{2}-E_{n l}^{2}\right)-2\left(\begin{array}{c}
E_{n l} V_{\mathrm{mcy}}(r) \\
+M S_{\mathrm{mcy}}(r)
\end{array}\right) \\
+V_{\mathrm{mcy}}^{2}(r)-S_{\mathrm{mcy}}^{2}(r)-\frac{l(l+1)}{r^{2}}
\end{array}\right) U_{n l}(r)=0 .
$$




\section{A. MAIRECHE}

The shorthand notation $E_{\mathrm{eff}}^{\mathrm{mcy}}=M^{2}-E_{n l}^{2}$ and $V_{\mathrm{eff}}^{\mathrm{mcy}}(r)=2\left(E_{n l} V_{\mathrm{mcy}}(r)+M S_{\mathrm{mcy}}(r)\right)-V_{\mathrm{mcy}}^{2}(r)+S_{\mathrm{mcy}}^{2}(r)+\frac{l(l+1)}{r^{2}}$, we obtain the following second-order Schrödinger-like equation:

$$
\left(\frac{d^{2}}{d r^{2}}-\left(E_{\mathrm{eff}}^{\mathrm{mcy}}+V_{\mathrm{eff}}^{\mathrm{mcy}}(r)\right)\right) U_{n l}(r)=0 .
$$

When the vector potential is equal to the scalar potential $V_{\mathrm{mp}}(r)=S_{\mathrm{mp}}(r)$, the effective potential of the ManningRosen plus a class of Yukawa potentials reduces to the form:

$$
V_{\mathrm{eff}}^{\mathrm{mcy}}(r)=2\left(E_{n l}+M\right)\left(\frac{1}{2 M b^{2}}\left[\frac{\eta(\eta-1) e^{-2 r / b}}{\left(1-e^{-r / b}\right)^{2}}-\frac{A e^{-r / b}}{1-e^{-r / b}}\right]-\frac{V_{0} e^{-\delta r}}{r}-\frac{V_{0}^{\prime} e^{-2 \delta r}}{r^{2}}\right)+\frac{l(l+1)}{r^{2}} .
$$

A. I. Ahmadov et al. [32] derived analytical expressions for the wave function and the corresponding energy values for the vector and scalar Manning-Rosen plus a class of Yukawa potentials using the linear combination of ManningRosen and a class of Yukawa potentials using both the Nikiforov-Uvarov, SUSYQM methods and the approximation scheme proposed by Greene and Aldrich as follows:

$$
\begin{gathered}
\Psi(r, \theta, \varphi)=\frac{C_{n l}}{r} s^{\epsilon_{n l}}(1-s)^{k_{n l}} \frac{\Gamma\left(n+2 \varepsilon_{n l}+1\right)}{n ! \Gamma\left(2 \varepsilon_{n l}+1\right)}{ }_{2} F_{1}\left(-n, 2 \varepsilon_{n l}+2 k_{n l}+n ; 1+2 \varepsilon_{n l} ; s\right) Y_{l}^{m}(\theta, \varphi), \\
M^{2}-E_{n l}^{2}=\delta^{2}\left[\frac{\beta_{n l}^{2}-l(l+1)-1 / 2-n(n+1)}{n+1 / 2+\sqrt{1 / 4+\alpha_{n l}^{2}+l(l+1)}}-\frac{(2 n+1) \sqrt{1 / 4+\alpha_{n l}^{2}+l(l+1)}}{n+1 / 2+\sqrt{1 / 4+\alpha_{n l}^{2}+l(l+1)}}\right]^{2},
\end{gathered}
$$

where $s=e^{-2 \delta r}, \varepsilon_{n l}=\frac{\sqrt{M^{2}-E_{n l}^{2}}}{2 \delta}, k_{n l}=1 / 2+\sqrt{1 / 4+\alpha_{n l}^{2}+l(l+1)}, \alpha_{n l}^{2}=\frac{E_{n l}+M}{2 \delta} V_{014}, V_{014}=\frac{2 \delta^{2} \eta(\eta-1)}{M}, \beta_{n l}^{2}=$ $\frac{E_{n l}+M}{2 \delta} V_{023}, V_{023}=\frac{2 \delta^{2} A}{M}+2 \delta V_{0}$, while ${ }_{2} F_{1}\left(-n, 2 \varepsilon_{n l}+2 k_{n l}+n ; 1+2 \varepsilon_{n l} ; s\right)$ are the hypergeometric polynomials and the normalization constant is given by [32]:

$$
C_{n l}=\sqrt{\frac{2 \delta n !\left(\varepsilon_{n l}+k_{n l}+n\right) \Gamma\left(2 \varepsilon_{n l}+2 k_{n l}+n\right) \Gamma\left(2 \varepsilon_{n l}+1\right)}{\left(k_{n l}+n\right) \Gamma\left(2 \varepsilon_{n l}+n+1\right) \Gamma\left(2 k_{n l}+n\right)}} .
$$

\section{THE SOLUTION OF DRKGE UNDER THE DVSMCY-PS MODEL IN RNCQM SYMMETRIES}

\section{A. Review of Bopp's shift method}

At the beginning of this section, we shall give and define a formula of the deformed equal vector scalar Manning-Rosen plus a class of Yukawa potentials in the symmetries of relativistic noncommutative threedimensional real space RNCQM symmetries. To achieve this goal, it is useful to write the DKGE by applying the notion of the Weyl-Moyal star product, which was seen previously in Eqs. (1.3), (1.4) and (1.5), to the differential equation that is satisfied by the radial wave function $U_{n l}(r)$ in Eq. (2.3). Thus, the radial wave function in RNCQM symmetries becomes as follows [72-79]:

$$
\left(\frac{d^{2}}{d r^{2}}-\left(E_{\mathrm{eff}}^{\mathrm{mcy}}+V_{\mathrm{eff}}^{\mathrm{mcy}}(r)\right)\right) * U_{n l}(r)=0 .
$$

It is established extensively in the literature and in a basic text $[64,75-80]$ that the star products can be simplified from Bopp's shift method. Physicist Fritz Bopp was the first to consider pseudo-differential operators obtained from a symbol by the quantization rules $x \rightarrow$ $x-\frac{i}{2} \frac{\partial}{\partial p}$ and $p \rightarrow p+\frac{i}{2} \frac{\partial}{\partial x}$ instead of the ordinary correspondence $x \rightarrow x$ and $p \rightarrow \frac{i}{2} \frac{\partial}{\partial x}$ [80, 81]. In physics literature, this is known as Bopp's shifts. This quantization procedure is called the Bopp quantization. It is known to the specialists that Bopp's shift method [64, 79, 80] has been applied effectively and has succeeded in simplifying the three basic equations: DSE, DKGE, and the deformed Dirac equation (DDE) with the notion of star product, to the Schrödinger equation (SE), KGE, and Dirac equation (DE) with the notion of ordinary product, respectively. Thus, Bopp's shift method is based on reducing second-order linear differential equations of DSE, DKGE, and DDE with star product to secondorder linear differential equations of SE, KGE, and DE without star product with simultaneous translation in the space-space. The CNCCRs with star product in Eqs. (1.3) and (1.4) become new CNCCRs without the notion of star product as follows (see, e.g., [41-51]):

$$
\begin{aligned}
& {\left[\hat{x}_{\mu}^{\mathrm{S}}, \hat{p}_{\nu}^{\mathrm{S}}\right]=\left[\hat{x}_{\mu}^{\mathrm{H}}, \hat{p}_{\nu}^{\mathrm{H}}\right]=\left[\hat{x}_{\mu}^{\mathrm{I}}, \hat{p}_{\nu}^{\mathrm{I}}\right]=i \hbar_{\mathrm{eff}} \delta_{\mu \nu},} \\
& {\left[\hat{x}_{\mu}^{\mathrm{S}}, \hat{x}_{\nu}^{\mathrm{S}}\right]=\left[\hat{x}_{\mu}^{\mathrm{H}}, \hat{x}_{\nu}^{\mathrm{H}}\right]=\left[\hat{x}_{\mu}^{\mathrm{I}}, \hat{x}_{\nu}^{\mathrm{I}}\right]=i \theta_{\mu \nu} .}
\end{aligned}
$$


The generalized positions and momentum coordinates $\left(\hat{x}_{\mu}^{\mathrm{S}}, \hat{x}_{\mu}^{\mathrm{H}}, \hat{x}_{\mu}^{\mathrm{I}}\right)$ and $\left(\hat{p}_{\mu}^{\mathrm{S}}, \hat{p}_{\mu}^{\mathrm{H}}, \hat{p}_{\mu}^{\mathrm{I}}\right)$ in the symmetries of RNCQM are defined in terms of the corresponding coordinates in the symmetries of RQM $\left(x_{\mu}^{\mathrm{S}}, x_{\mu}^{\mathrm{H}}, x_{\mu}^{\mathrm{I}}\right)$ and $\left(p_{\mu}^{\mathrm{S}}, p_{\mu}^{\mathrm{H}}, p_{\mu}^{\mathrm{I}}\right)$ via, respectively [40-50]:

$$
\begin{aligned}
& \hat{x}_{\mu}^{(\mathrm{S}, \mathrm{H}, \mathrm{I})}=x_{\mu}^{(\mathrm{S}, \mathrm{H}, \mathrm{I})}-\sum_{\nu=1}^{3} \frac{\theta_{\mu \nu}}{2} p_{\nu}^{(\mathrm{S}, \mathrm{H}, \mathrm{I})}, \\
& \hat{p}_{\mu}^{(\mathrm{S}, \mathrm{H}, \mathrm{I})}=p_{\mu}^{(\mathrm{S}, \mathrm{H}, \mathrm{I})} .
\end{aligned}
$$

This allows us to find the operator $r_{\text {nc }}^{2}$ equal $r^{2}-\mathbf{L} \Theta$ (see the Introduction) in NCQM symmetries [68, 69].

\section{B. New effective potential in RNCQM symmetries}

According to the Bopp shift method, Eq. (3.1) becomes similar to the following like the Schrödinger equation (without the notions of star product):

$$
\left(\begin{array}{c}
\frac{d^{2}}{d r^{2}}-\left(M^{2}-E_{n l}^{2}\right)-\frac{l(l+1)}{r_{\mathrm{nc}}^{2}} \\
-2 V_{\mathrm{mcy}}\left(r_{\mathrm{nc}}\right)\left(E_{n l}+M\right)
\end{array}\right) U_{n l}(r)=0
$$

The new operators $V_{\mathrm{mcy}}\left(r_{\mathrm{nc}}\right)$ and $\frac{l(l+1)}{r_{\mathrm{nc}}^{2}}$ are expressed as in RNCQM symmetries as follows:

$$
\left\{\begin{array}{c}
V_{\mathrm{mcy}}\left(r_{\mathrm{nc}}\right)=\frac{1}{2 M b^{2}}\left[\frac{\eta(\eta-1) e^{-2 r / b}}{\left(1-e^{-r / b}\right)^{2}}-\frac{A e^{-r / b}}{1-e^{-r / b}}\right]-\frac{V_{0} e^{-\delta r}}{r}-\frac{V_{0}^{\prime} e^{-2 \delta r}}{r^{2}}-\frac{\partial V_{\mathrm{msy}}(r)}{\partial r} \frac{\mathbf{L} \Theta}{2 r}+O\left(\Theta^{2}\right) \\
\frac{l(l+1)}{r_{\mathrm{nc}}^{2}}=\frac{l(l+1)}{r^{2}}+\frac{l(l+1)}{r^{4}} \mathbf{L} \Theta+O\left(\Theta^{2}\right)
\end{array} .\right.
$$

So we can rewrite:

$$
V_{\mathrm{mcy}}\left(r_{\mathrm{nc}}\right)\left(E_{n l}+M\right)=V_{\mathrm{mcy}}(r)\left(E_{n l}+M\right)-\frac{E_{n l}+M}{2 r} \frac{\partial V_{\mathrm{msy}}(r)}{\partial r} \mathbf{L} \Theta+O\left(\Theta^{2}\right) .
$$

Moreover, to illustrate the above equation in a simple mathematical way and attractive form, it is useful to introduce the following symbol, $V_{\mathrm{nc}-\mathrm{eff}}^{\mathrm{mcy}}(r)$, thus the radial Eq. (3.6) becomes:

$$
\left(\frac{d^{2}}{d r^{2}}-\left(E_{\mathrm{eff}}^{\mathrm{mcy}}+V_{\mathrm{nc}-\mathrm{eff}}^{\mathrm{mcy}}(r)\right)\right) U_{n l}(r)=0
$$

with:

$$
V_{\text {nc-eff }}^{\text {mcy }}(r)=V_{\text {eff }}^{\text {mcy }}(r)+V_{\text {pert }}^{\text {mcy }}(r) \text {. }
$$

Moreover, $V_{\text {pert }}^{\text {mcy }}(r)$ is given by the following relation:

$$
\begin{aligned}
V_{\text {pert }}^{\text {mcy }}(r) & =\frac{l(l+1)}{r^{4}} \mathbf{L} \Theta-\frac{E_{n l}+M}{r} \frac{\partial V_{\text {msy }}(r)}{\partial r} \mathbf{L} \Theta \\
& +O\left(\Theta^{2}\right.
\end{aligned}
$$

It should be noted that Eq. (2.3) with the ManningRosen plus a class of Yukawa potentials can be exactly solved for $l=0$, but for the case $l \neq 0$, A. I. Ahmadov et al. had approximatively solved the equation using Eq. (2.5) within the Greene-Aldrich approximation scheme in RQM symmetries. In the new form of radial Schrödinger-like equation written in Eq. (3.9), we have terms including $\frac{1}{r}, \frac{1}{r^{4}}$ and other Coulombic-like terms, which make this equation impossible to solve analytically for $l=0$ and $l \neq 0$; it can only be solved approximately. From this point of view, we can consider the improved approximation of the centrifugal term proposed by M. Badawi et al. [82]. This method proved its power and efficiency when compared with the Greene and Aldrich approximation [6]. The approximations type suggested by (Greene and Aldrich) and Dong et al. for a short-range potential is an excellent approximation to the centrifugal term and allows us to get a second order solvable differential equation. Unlike the approximation used in the previous works [19, 30-32, 45, 49, 50, 79], here we use:

$$
\frac{1}{r^{2}} \approx \frac{4 \delta^{2} e^{-2 \delta r}}{\left(1-e^{-2 \delta r}\right)^{2}}=\frac{4 \delta^{2} s}{(1-s)^{2}} .
$$

It is important to mention here that the above approximations are valid when $\delta r \ll 1$. This allows us to obtain:

$$
\frac{1}{r} \approx \frac{2 \delta e^{-\delta r}}{1-e^{-2 \delta r}}=\frac{2 \delta s^{1 / 2}}{1-s} .
$$

Now we rewrite the Manning-Rosen potential plus a class of Yukawa potentials under the assumption of $\frac{1}{b}=$ $2 \delta$ as follows:

$$
\begin{aligned}
V_{\mathrm{mcy}}(r) & =\frac{1}{2 M b^{2}}\left[\frac{\eta(\eta-1) e^{-4 \delta r}}{\left(1-e^{-2 \delta r}\right)^{2}}-\frac{A e^{-\delta r}}{1-e^{-\delta r}}\right] \\
& -\frac{V_{0} e^{-\delta r}}{r}-\frac{V_{0}^{\prime} e^{-2 \delta r}}{r^{2}} .
\end{aligned}
$$




\section{A. MAIRECHE}

After straightforward calculations, we obtain $\frac{\partial V_{\mathrm{msy}}(r)}{\partial r}$ as follows:

$$
\begin{aligned}
& \frac{\partial V_{\mathrm{msy}}(r)}{\partial r} \\
& =-\frac{8 \delta^{3}}{M} \eta(\eta-1)\left(\frac{e^{-4 \delta r}}{\left(1-e^{-2 \delta r}\right)^{2}}+\frac{e^{-6 \delta r}}{\left(1-e^{-2 \delta r}\right)^{2}}\right) \\
& +\frac{4 \delta^{3} A}{M} \frac{e^{-2 \delta r}}{1-e^{-2 \delta r}}+\frac{4 \delta^{3} A}{M} \frac{e^{-4 \delta r}}{\left(1-e^{-2 \delta r}\right)^{2}} \\
& +\frac{V_{0} e^{-\delta r}}{r}+\frac{V_{0} e^{-\delta r}}{r^{2}}+2 \delta \frac{V_{0}^{\prime} e^{-2 \delta r}}{r^{2}}+2 \delta \frac{V_{0}^{\prime} e^{-2 \delta r}}{r^{3}} .
\end{aligned}
$$

We apply the approximations of Greene and Aldrich to the expression $\frac{\partial V_{\mathrm{msy}}(r)}{\partial r}$ and get the following formula

$$
\begin{aligned}
\frac{\partial V_{\mathrm{msy}}(r)}{\partial r} & =\lambda_{1} \frac{s^{2}}{(1-s)^{2}}+\lambda_{2} \frac{s^{3}}{(1-s)^{3}}+\lambda_{3} \frac{s}{1-s} \\
& +\lambda_{4} \frac{s^{3 / 2}}{(1-s)^{2}}+\lambda_{5} \frac{s^{5 / 2}}{(1-s)^{3}}
\end{aligned}
$$

with $\lambda_{1}=8 \delta^{3} V_{0}+\lambda_{2}, \lambda_{2}=-\frac{8 \delta^{3}}{M} \eta(\eta-1), \lambda_{3}=\frac{8 \delta^{3} A}{M}+$ $2 V_{0} \delta^{2}, \lambda_{4}=4 V_{0} \delta^{2}$ and $\lambda_{5}=16 V_{0}^{\prime} \delta^{3}$. This allows us to write the second part of Eq. (3.11) as follows:

$$
\begin{aligned}
& \frac{E_{n l}+M}{r} \frac{\partial V_{\mathrm{msy}}(r)}{\partial r}=2 \delta\left(E_{n l}+M\right) \\
& \times\left(\begin{array}{c}
\lambda_{1} \frac{s^{5 / 2}}{(1-s)^{3}}+\lambda_{2} \frac{s^{7 / 2}}{(1-s)^{4}} \\
+\lambda_{3} \frac{s^{3 / 2}}{(1-s)^{2}}+\lambda_{4} \frac{s^{2}}{(1-s)^{3}}+\lambda_{5} \frac{s^{3}}{(1-s)^{4}}
\end{array}\right) .
\end{aligned}
$$

By substituting Eq. (3.17) into Eq. (3.11), we find the perturbed effective potential $V_{\text {pert }}^{\text {mcy }}(r)$ generated from noncommutativity properties of space-space in the symmetries of RNCQM as follows:

$$
\begin{aligned}
& V_{\text {pert }}^{\text {mcy }}(r)=\frac{16 \delta^{4} l(l+1) s^{2}}{(1-s)^{4}} \mathbf{L} \Theta-2 \delta\left(E_{n l}+M\right) \\
& \times\left(\begin{array}{c}
\lambda_{1} \frac{s^{5 / 2}}{(1-s)^{3}}+\lambda_{2} \frac{s^{7 / 2}}{(1-s)^{4}} \\
+\lambda_{3} \frac{s^{3 / 2}}{(1-s)^{2}}+\lambda_{4} \frac{s^{2}}{(1-s)^{3}}+\lambda_{5} \frac{s^{3}}{(1-s)^{4}}
\end{array}\right) \mathbf{L} \Theta+O\left(\Theta^{2}\right) .
\end{aligned}
$$

We have applied the approximations of Greene and Aldrich to the term $\frac{l(l+1)}{r^{4}}$. The Manning-Rosen potential plus a class of Yukawa potentials is extended by including new terms proportional to the radial terms $\frac{s^{2}}{(1-s)^{4}}, \frac{s^{5 / 2}}{(1-s)^{3}}, \frac{s^{7 / 2}}{(1-s)^{4}}, \frac{s^{3 / 2}}{(1-s)^{2}}, \frac{s^{2}}{(1-s)^{3}}$, and $\frac{s^{3}}{(1-s)^{4}}$ to become the deformed Manning-Rosen potential plus a class of Yukawa potentials in RNCQM symmetries. The generated new effective potential $V_{\text {nç-eff }}^{\text {mcy }}(r)$ is also proportional to the infinitesimal vector $\vec{\Theta}$. This allows us to consider $V_{\text {pert }}^{\text {mcy }}(r)$ to be a perturbation potential compared with the main potential $V_{\text {eff }}^{\text {mcy }}(r)$ (parent potential operator in the symmetries of RNCQM, that is, the inequality $V_{\text {pert }}^{\text {mcy }}(r) \ll V_{\text {eff }}^{\text {mcy }}(r)$ has been achieved. That is, all the physical justifications for applying the time-independent perturbation theory become satisfied. This allows us to give a complete prescription for determining the energy level of the generalized excited states.

\section{The expectation values in RNCQM symmetries}

In this sub-section, we want to apply the perturbative theory. In the case of RNCQM, we find the expectation values of the radial terms $\frac{s^{2}}{(1-s)^{4}}, \frac{s^{5 / 2}}{(1-s)^{3}}, \frac{s^{7 / 2}}{(1-s)^{4}}, \frac{s^{3 / 2}}{(1-s)^{2}}$, $\frac{s^{2}}{(1-s)^{3}}$, and $\frac{s^{3}}{(1-s)^{4}}$ and take into account the wave function which we have seen previously in Eq. (2.5). Thus, after straightforward calculations, we obtain the following results:

$$
\begin{aligned}
& \left\langle\frac{s^{2}}{(1-s)^{4}}\right\rangle_{(n l m)}=C_{n l}^{n} \int_{0}^{+\infty} s^{2 \epsilon_{n l}}(1-s)^{2 k_{n l}} \quad\left[{ }_{2} F_{1}\left(-n, 2 \varepsilon_{n l}+2 k_{n l}+n ; 1+2 \varepsilon_{n l} ; s\right)\right]^{2} \frac{s^{2} d r}{(1-s)^{4}}, \\
& \left\langle\frac{s^{5 / 2}}{(1-s)^{3}}\right\rangle_{(n l m)}=C_{n l}^{n} \int_{0}^{+\infty} s^{2 \epsilon_{n l}}(1-s)^{2 k_{n l}}\left[{ }_{2} F_{1}\left(-n, 2 \varepsilon_{n l}+2 k_{n l}+n ; 1+2 \varepsilon_{n l} ; s\right)\right]^{2} \frac{s^{5 / 2} d r}{(1-s)^{3}}, \\
& \left\langle\frac{s^{7 / 2}}{(1-s)^{4}}\right\rangle_{(n l m)}=C_{n l}^{n} \int_{0}^{+\infty} s^{2 \epsilon_{n l}}(1-s)^{2 k_{n l}}\left[{ }_{2} F_{1}\left(-n, 2 \varepsilon_{n l}+2 k_{n l}+n ; 1+2 \varepsilon_{n l} ; s\right)\right]^{2} \frac{s^{7 / 2} d r}{(1-s)^{4}}, \\
& \left\langle\frac{s^{3 / 2}}{(1-s)^{2}}\right\rangle_{(n l m)}=C_{n l}^{n} \int_{0}^{+\infty} s^{2 \epsilon_{n l}}(1-s)^{2 k_{n l}}\left[{ }_{2} F_{1}\left(-n, 2 \varepsilon_{n l}+2 k_{n l}+n ; 1+2 \varepsilon_{n l} ; s\right)\right]^{2} \frac{s^{3 / 2} d r}{(1-s)^{2}},
\end{aligned}
$$




$$
\begin{aligned}
& \left\langle\frac{s^{2}}{(1-s)^{3}}\right\rangle_{(n l m)}=C_{n l}^{n} \int_{0}^{+\infty} s^{2 \epsilon_{n l}}(1-s)^{2 k_{n l}} \quad\left[{ }_{2} F_{1}\left(-n, 2 \varepsilon_{n l}+2 k_{n l}+n ; 1+2 \varepsilon_{n l} ; s\right)\right]^{2} \frac{s^{2} d r}{(1-s)^{3}}, \\
& \left\langle\frac{s^{3}}{(1-s)^{4}}\right\rangle_{(n l m)}=C_{n l}^{n} \int_{0}^{+\infty} s^{2 \epsilon_{n l}}(1-s)^{2 k_{n l}}\left[{ }_{2} F_{1}\left(-n, 2 \varepsilon_{n l}+2 k_{n l}+n ; 1+2 \varepsilon_{n l} ; s\right)\right]^{2} \frac{s^{3} d r}{(1-s)^{4}},
\end{aligned}
$$

with $C_{n l}^{n}=\left[C_{n l} \frac{\Gamma\left(n+2 \varepsilon_{n l}+1\right)}{n ! \Gamma\left(2 \varepsilon_{n l}+1\right)}\right]^{2}$. We have used useful abbreviations $\langle D\rangle_{(n l m)}=\langle n, l, m D n, l, m\rangle$ to avoid the extra burden of writing equations. It should be noted that the above equations are a physical translation of the first-order energy corrections that can generally be obtained by applying the following relationship known in the literature [83]:

$$
\left\langle V_{\text {pert }}^{\mathrm{mcy}}(r)\right\rangle_{(n l m)}=\int_{0}^{+\infty} \Psi^{*}(r, \theta, \varphi) V_{\mathrm{pert}}^{\mathrm{mcy}}(r) \Psi(r, \theta, \varphi) d^{3} r
$$

Here, $d^{3} r=r^{2} \sin (\theta) d \theta d \varphi d r$. Furthermore, we have applied the property of the spherical harmonics, which has the form $\int Y_{l}^{m}(\theta, \varphi) Y_{l^{\prime}}^{m^{\prime}}(\theta, \varphi) \sin \theta d \theta d \varphi=\delta_{l l^{\prime}} \delta_{m m^{\prime}}$. We have $s=e^{-2 \delta r}$, this allows us to obtain $d r=-\frac{1}{2 \delta} \frac{d s}{s}$. From the asymptotic behavior of $s=e^{-2 \delta r}$ when $r \rightarrow 0(s \rightarrow+1)$ and when $r \rightarrow+\infty(s \rightarrow 0)$, this allows us to reformulate Eqs. (3.19)-(3.24) as follows:

$$
\begin{aligned}
& \left\langle\frac{s^{2}}{(1-s)^{4}}\right\rangle_{(n l m)}=N_{\text {new }}^{\text {mcp }} \int_{0}^{+1} s^{2 \epsilon_{n l}+1}(1-s)^{2 k_{n l}-4}\left[{ }_{2} F_{1}\left(-n, 2 \varepsilon_{n l}+2 k_{n l}+n ; 1+2 \varepsilon_{n l} ; s\right)\right]^{2} d s \\
& \left\langle\frac{s^{5 / 2}}{(1-s)^{3}}\right\rangle_{(n l m)}=N_{\text {new }}^{\text {mcp }} \int_{0}^{+1} s^{2 \epsilon_{n l}+3 / 2}(1-s)^{2 k_{n l}-3}\left[{ }_{2} F_{1}\left(-n, 2 \varepsilon_{n l}+2 k_{n l}+n ; 1+2 \varepsilon_{n l} ; s\right)\right]^{2} d s \\
& \left\langle\frac{s^{7 / 2}}{(1-s)^{4}}\right\rangle_{(n l m)}=N_{\text {new }}^{\text {mcp }} \int_{0}^{+1} s^{2 \epsilon_{n l}+5 / 2}(1-s)^{2 k_{n l}-4}\left[{ }_{2} F_{1}\left(-n, 2 \varepsilon_{n l}+2 k_{n l}+n ; 1+2 \varepsilon_{n l} ; s\right)\right]^{2} d s, \\
& \left\langle\frac{s^{3 / 2}}{(1-s)^{2}}\right\rangle_{(n l m)}=N_{\text {new }}^{\text {mcp }} \int_{0}^{+1} s^{2 \epsilon_{n l}+1 / 2}(1-s)^{2 k_{n l}-2}\left[{ }_{2} F_{1}\left(-n, 2 \varepsilon_{n l}+2 k_{n l}+n ; 1+2 \varepsilon_{n l} ; s\right)\right]^{2} d s \\
& \left\langle\frac{s^{2}}{(1-s)^{3}}\right\rangle_{(n l m)}=N_{\text {new }}^{\text {mcp }} \int_{0}^{+1} s^{2 \epsilon_{n l}+1}(1-s)^{2 k_{n l}-3}\left[{ }_{2} F_{1}\left(-n, 2 \varepsilon_{n l}+2 k_{n l}+n ; 1+2 \varepsilon_{n l} ; s\right)\right]^{2} d s \\
& \left\langle\frac{s^{3}}{(1-s)^{4}}\right\rangle_{(n l m)}=N_{\text {new }}^{\text {mcp }} \int_{0}^{+1} s^{2 \epsilon_{n l}+2}(1-s)^{2 k_{n l}-4}\left[{ }_{2} F_{1}\left(-n, 2 \varepsilon_{n l}+2 k_{n l}+n ; 1+2 \varepsilon_{n l} ; s\right)\right]^{2} d s
\end{aligned}
$$

Here, $N_{\text {new }}^{\text {mcp }}=\frac{C_{n l}^{n}}{2 \delta}$. By using the same method as that proposed by Dong et al. [84] and applied by Zhang [85], we calculate the integrals in Eqs. (3.25)-(3.30). With the help of the special integral formula,

$$
\int_{0}^{+1} s^{\xi-1}(1-s)^{\sigma-1}\left[{ }_{2} F_{1}\left(c_{1}, c_{2} ; c_{3} ; s\right)\right]^{2} d s=\frac{\Gamma(\xi) \Gamma(\sigma)}{\Gamma(\xi+\sigma)}{ }_{3} F_{2}\left(c_{1}, c_{2}, \sigma ; c_{3}, \sigma+\xi ; 1\right),
$$




\section{A. MAIRECHE}

where ${ }_{3} F_{2}\left(c_{1}, c_{2}, \sigma ; c_{3}, \sigma+\xi ; 1\right)$ is obtained from the generalized hypergeometric function ${ }_{p} F_{q}\left(\alpha_{1}, \alpha_{2}, \ldots, \alpha_{p} ; \beta_{1}, \beta_{2}, \ldots, \beta_{q} ; 1\right)$ for $p=3$ and $q=2$ while $\Gamma(\sigma)$ denotes the usual Gamma function.

We obtain from Eqs. (3.25)-(3.30) the results:

$$
\begin{aligned}
& \left\langle\frac{s^{2}}{(1-s)^{4}}\right\rangle_{(n l m)}=N_{\text {new }}^{\text {mcp }} \frac{\Gamma\left(2 \epsilon_{n l}+2\right) \Gamma\left(2 k_{n l}-3\right)}{\Gamma(\Omega-1)}{ }_{3} F_{2}\left(-n, \Omega+n, 2 k_{n l}-3 ; 1+2 \varepsilon_{n l}, \Omega-1 ; 1\right), \\
& \left\langle\frac{s^{5 / 2}}{(1-s)^{3}}\right\rangle_{(n l m)}=N_{\text {new }}^{\text {mcp }} \frac{\Gamma\left(2 \epsilon_{n l}+5 / 2\right) \Gamma\left(2 k_{n l}-2\right)}{\Gamma(\Omega+1 / 2)}{ }_{3} F_{2}\left(-n, \Omega+n, 2 k_{n l}-2 ; 1+2 \varepsilon_{n l}, \Omega+1 / 2 ; 1\right), \\
& \left\langle\frac{s^{7 / 2}}{(1-s)^{4}}\right\rangle_{(n l m)}=N_{\text {new }}^{\text {mcp }} \frac{\Gamma\left(2 \epsilon_{n l}+7 / 2\right) \Gamma\left(2 k_{n l}-3\right)}{\Gamma(\Omega+1 / 2)}{ }_{3} F_{2}\left(-n, \Omega+n, 2 k_{n l}-3 ; 1+2 \varepsilon_{n l}, \Omega+1 / 2 ; 1\right) \\
& \left\langle\frac{s^{3 / 2}}{(1-s)^{2}}\right\rangle_{(n l m)}=N_{\text {new }}^{\text {mcp }} \frac{\Gamma\left(2 \epsilon_{n l}+3 / 2\right) \Gamma\left(2 k_{n l}-1\right)}{\Gamma(\Omega+1 / 2)}{ }_{3} F_{2}\left(-n, \Omega+n, 2 k_{n l}-1 ; 1+2 \varepsilon_{n l}, \Omega+1 / 2 ; 1\right), \\
& \left\langle\frac{s^{2}}{(1-s)^{3}}\right\rangle_{(n l m)}=N_{\text {new }}^{\text {mcp }} \frac{\Gamma\left(2 \epsilon_{n l}+2\right) \Gamma\left(2 k_{n l}-2\right)}{\Gamma(\Omega)}{ }_{3} F_{2}\left(-n, \Omega+n, 2 k_{n l}-2 ; 1+2 \varepsilon_{n l}, \Omega ; 1\right), \\
& \left\langle\frac{s^{3}}{(1-s)^{4}}\right\rangle_{(n l m)} N_{\text {new }}^{\text {mcp }} \frac{\Gamma\left(2 \epsilon_{n l}+3\right) \Gamma\left(2 k_{n l}-3\right)}{\Gamma(\Omega)}{ }_{3} F_{2}\left(-n, \Omega+n, 2 k_{n l}-3 ; 1+2 \varepsilon_{n l}, \Omega ; 1\right),
\end{aligned}
$$

where $\Omega\left(\delta, \eta, b, A, V_{0}, V_{0}^{\prime}\right)=2 \epsilon_{n l}\left(\delta, \eta, b, A, V_{0}, V_{0}^{\prime}\right)+2 k_{n l}\left(\delta, \eta, b, A, V_{0}, V_{0}^{\prime}\right)$.

\section{The energy shift for the deformed equal vector scalar Manning-Rosen plus a class of Yukawa potentials in RNCQM symmetries}

The global relativistic energy shift GRES for the deformed equal vector scalar Manning-Rosen plus a class of Yukawa potentials in RNCQM symmetries is composed of three principal parts. The first one is produced from the effect of the generated spin-orbit effective potential. This effective potential is obtained by replacing the coupling of the angular momentum operator and the noncommutative vector $\mathbf{L} \Theta$ with the new equivalent coupling $\Theta \mathbf{L S}$ (with $\Theta^{2}=\Theta_{12}^{2}+\Theta_{23}^{2}+$ $\Theta_{13}^{2}$ ). This degree of freedom came considering that the infinitesimal noncommutative vector $\Theta$ is arbitrary. We have chosen it to a parallel of the spin $\mathbf{S}$ of the diatomic molecules under the deformed equal vector scalar Manning-Rosen plus a class of Yukawa potentials. Furthermore, we replace the new spin-orbit coupling $\Theta \mathbf{L S}$ with the corresponding physical form
$(\Theta / 2) \mathbf{G}^{2}$, with $\mathbf{G}^{2}=\mathbf{J}^{2}-\mathbf{L}^{2}-\mathbf{S}^{2}$. Moreover, in quantum mechanics, the operators $\widehat{H}_{r n c}^{\text {mcy }}, \mathbf{J}^{2}, \mathbf{L}^{2}, \mathbf{S}^{2}$, and $\mathbf{J}_{z}$ form a complete set of conserved physics quantities, the eigenvalues of the operator $\mathbf{G}^{2}$ equal the values $k(j, l, s)=$ $[j(j+1)-l(l+1)-s(s+1)] / 2$, with $|l-s| \leq j \leq$ $|l+s|$. As a direct consequence, the partial energy shift $\Delta E_{\mathrm{mcy}}^{\mathrm{so}}\left(n, \delta, \eta, b, A, V_{0}, V_{0}^{\prime}, \Theta, j, l, s\right)$ due to the perturbed effective potential $V_{\text {pert }}^{\text {mcy }}(r)$ produced for the $n^{\text {th }}$ excited state, in RNCQM symmetries is as follows:

$$
\begin{aligned}
& \Delta E_{\mathrm{mcy}}^{\mathrm{so}}\left(n, \delta, \eta, b, A, V_{0}, V_{0}^{\prime}, \Theta, j, l, s\right) \\
& =\Theta(j(j+1)-l(l+1)-s(s+1)) \\
& \times\langle\Xi\rangle_{(n l m)}^{\mathrm{RMCY}}\left(n, \delta, \eta, b, A, V_{0}, V_{0}^{\prime}\right) .
\end{aligned}
$$

The global expectation value $\langle\Xi\rangle_{(n l m)}^{\mathrm{RMCY}}(n, \delta, \eta, b, A$, $\left.V_{0}, V_{0}^{\prime}\right)$ is determined from the following expression: 


$$
\begin{aligned}
& \langle\Xi\rangle_{(n l m)}^{\mathrm{RMCY}}\left(n, \delta, \eta, b, A, V_{0}, V_{0}^{\prime}\right)=16 \delta^{4} l(l+1)\left\langle\frac{s^{2}}{(1-s)^{4}}\right\rangle_{(n l m)}-2 \delta\left(E_{n l}+M\right) \lambda_{1}\left\langle\frac{s^{5 / 2}}{(1-s)^{3}}\right\rangle_{(n l m)} \\
& -2 \delta\left(E_{n l}+M\right)\left(+\lambda_{2}\left\langle\frac{s^{7 / 2}}{(1-s)^{4}}\right\rangle_{(n l m)} \lambda_{3}\left\langle\frac{s^{3 / 2}}{(1-s)^{2}}\right\rangle_{(n l m)}+\lambda_{4}\left\langle\frac{s^{2}}{(1-s)^{3}}\right\rangle_{(n l m)}+\lambda_{5}\left\langle\frac{s^{3}}{(1-s)^{4}}\right\rangle_{(n l m)}\right) .
\end{aligned}
$$

The second part of the new effective potential $V_{\text {nc-eff }}^{\text {mcy }}(r)$ is obtained from the magnetic effect of perturbative effective potential $V_{\text {pert }}^{\text {mcy }}(r)$ under the deformed equal vector scalar Manning-Rosen plus a class of Yukawa potentials. This effective potential is achieved when we replace both $\mathbf{L} \Theta$ and $\Theta_{12}$ with $\left(\sigma B L_{z}\right.$ and $\sigma B)$, respectively; here, $B$ and $\sigma$ symbolize the intensity of the magnetic field induced by the effect of deformation of space-space geometry and a new infinitesimal noncommutativity parameter, so that the physical unit of the original noncommutativity parameter $\Theta_{12}$ is [length $]^{2}$ is the same unit of $\sigma B$. We also need to apply $\left\langle n^{\prime}, l^{\prime}, m^{\prime} L_{z} n, l, m\right\rangle=m \delta_{m^{\prime} m} \delta_{l^{\prime} l} \delta_{n^{\prime} n}\left(-l^{\prime} \leq m^{\prime} \leq l\right.$ and $-l \leq m \leq l$ ). All of this data allows for the discovery of the new energy shift $\Delta E_{\mathrm{mcy}}^{\mathrm{mag}}\left(n, \delta, \eta, b, A, V_{0}, V_{0}^{\prime}, \sigma, m\right)$ due to the perturbed Zeeman effect, which is created by the influence of the deformed equal vector scalar Manning-Rosen plus a class of Yukawa potentials for the $n^{\text {th }}$ excited-state in RNCQM symmetries, as follows:

$$
\begin{aligned}
& \Delta E_{\mathrm{mcy}}^{\mathrm{mag}}\left(n, \delta, \eta, b, A, V_{0}, V_{0}^{\prime}, \sigma, j, l, s\right) \\
& =\sigma B\langle\Xi\rangle_{(n l m)}^{\mathrm{RMCY}}\left(n, \delta, \eta, b, A, V_{0}, V_{0}^{\prime}\right) m .
\end{aligned}
$$

Now, for our purposes, we are interested in finding a new third automatically important symmetry for the deformed equal vector scalar Manning-Rosen plus a class of Yukawa potentials at zero temperature in RNCQM symmetries. This physical phenomenon is induced automatically from the influence of the perturbed effective potential $V_{\text {pert }}^{\text {mcy }}(r)$, which we have seen in Eq. (3.18). We discover these important physical phenomena when our studied system consists of non-interacting particles and is considered as the Fermi gas. It is formed from all the particles in their gaseous state $(\mathrm{HCl}, \mathrm{CH}, \mathrm{LiH}$, and $\mathrm{CO})$ undergoing rotation with angular velocity $\Omega$, if we make the following two simultaneous transformations to ensure that previous calculations are not repeated:

$$
\Theta \rightarrow \chi \boldsymbol{\Omega} \quad \text { and } \quad \mathbf{L} \Theta \rightarrow \chi \mathbf{L} \boldsymbol{\Omega}
$$

Here, $\chi$ is just infinitesimal real proportional constants. We can express the effective potential $V_{\text {pert }}^{\text {mcy-rot }}(r)$ which induced the rotational movements of the diatomic molecules as follows:

$$
\begin{aligned}
V_{\text {pert }}^{\text {mcy-rot }}(r) & =\frac{16 \delta^{4} l(l+1) \chi s^{2}}{(1-s)^{4}} \mathbf{L} \boldsymbol{\Omega}-2 \delta\left(E_{n l}+M\right) \\
& \times\left(\lambda_{1} \frac{s^{5 / 2}}{(1-s)^{3}}+\lambda_{2} \frac{s^{7 / 2}}{(1-s)^{4}}+\lambda_{3} \frac{s^{3 / 2}}{(1-s)^{2}}+\lambda_{4} \frac{s^{2}}{(1-s)^{3}}+\lambda_{5} \frac{s^{3}}{(1-s)^{4}}\right) \chi \mathbf{L} \boldsymbol{\Omega} .
\end{aligned}
$$

To simplify the calculations without compromising physical content, we choose the rotational velocity $\boldsymbol{\Omega}$ parallel to the $O z$ axis. Then we transform the spin-orbit coupling to the new physical phenomena as follows:

$$
\chi f(s) \mathbf{L} \boldsymbol{\Omega}=\chi f(s) \Omega L_{z}
$$

with

$$
f(s)=\frac{16 \delta^{4} l(l+1) s^{2}}{(1-s)^{4}}-2 \delta\left(E_{n l}+M\right)\left(\lambda_{1} \frac{s^{5 / 2}}{(1-s)^{3}}+\lambda_{2} \frac{s^{7 / 2}}{(1-s)^{4}}+\lambda_{3} \frac{s^{3 / 2}}{(1-s)^{2}}+\lambda_{4} \frac{s^{2}}{(1-s)^{3}}+\lambda_{5} \frac{s^{3}}{(1-s)^{4}}\right)
$$




\section{A. MAIRECHE}

All of this data allows for the discovery of the new energy shift $\Delta E_{\text {mcy }}^{\mathrm{f} \text {-rot }}\left(n, \delta, \eta, b, A, V_{0}, V_{0}^{\prime}, \chi, m\right)$ due to the perturbed Fermi gas effect $V_{\text {pert }}^{\text {mcy-rot }}(r)$, which is generated automatically by the influence of the deformed equal vector scalar Manning-Rosen plus a class of Yukawa potentials for the ground state and the first excited state in RNCQM symmetries as follows:

$$
\begin{aligned}
& \Delta E_{\mathrm{mcy}}^{\mathrm{f}-\mathrm{rot}}\left(n, \delta, \eta, b, A, V_{0}, V_{0}^{\prime}, \chi, m\right) \\
& =\chi\langle\Xi\rangle_{(n l m)}^{\mathrm{RMCY}}\left(n, \delta, \eta, b, A, V_{0}, V_{0}^{\prime}\right) \Omega m .
\end{aligned}
$$

It is worth mentioning that $\mathrm{K}$. Bencheikh et al. [86, 87] studied rotating isotropic and anisotropic harmonically confined ultra-cold Fermi gas in a two and threedimensional space at zero temperature, but in that study, the rotational term was added to the Hamiltonian operator, in contrast to our case, where this rotational term $\chi f(s) \mathbf{L} \boldsymbol{\Omega}$ automatically appears due to the large symmetries resulting from the deformation of spacephase.

\section{RESULTS AND DISCUSSION}

In this section, we summarize our obtained results $\Delta E_{\mathrm{mcy}}^{\mathrm{so}}\left(n, \delta, \eta, b, A, V_{0}, V_{0}^{\prime}, j, l, s\right), \Delta E_{\mathrm{mcy}}^{\mathrm{mag}}\left(n, \delta, \eta, b, A, V_{0}\right.$, $\left.V_{0}^{\prime}, m\right)$, and $\Delta E_{\text {mcy }}^{\mathrm{f}-\operatorname{rot}}\left(n, \delta, \eta, b, A, V_{0}, V_{0}^{\prime}, m\right)$ for the $n^{\text {th }}$ excited state due to the spin-orbit coupling, the modified Zeeman effect, and modified Fermi phenomena induced by $V_{\text {pert }}^{\text {mcy }}(r)$ due to the superposition princi- ple. This allows us to deduce the additive energy shift $\Delta E_{\mathrm{mcy}}^{\mathrm{tot}}\left(n, \delta, \eta, b, A, V_{0}, V_{0}^{\prime}, j, l, s, m\right)$ under the influence of DVSMCY-Ps in RNCQM symmetries as follows:

$$
\begin{aligned}
& \Delta E_{\mathrm{mcy}}^{\mathrm{tot}}\left(n, \delta, \eta, b, A, V_{0}, V_{0}^{\prime}, \Theta, \sigma, \chi, j, l, s, m\right) \\
& =\langle\Xi\rangle_{(n l m)}^{\mathrm{RMCY}}\left(n, \delta, \eta, b, A, V_{0}, V_{0}^{\prime}\right) \\
& \times(\Theta k(j, l, s)+\sigma B+m \chi \Omega m) .
\end{aligned}
$$

The above results present the global energy shift, which is generated with the effect of noncommutativity properties of space-space; it depended explicitly on the noncommutativity parameters $(\Theta, \sigma, \chi)$, the parameters of the equal vector scalar Manning-Rosen plus a class of Yukawa potentials $\left(\eta, b, A, V_{0}, V_{0}^{\prime}\right)$ in addition to the atomic quantum numbers $(j, l, s, m)$. We observed that the obtained global effective energy under DVSMCY-Ps has a carry unit of energy because it consists of the carrier of energy $\left(M^{2}-E_{n l}^{2}\right)$. In the symmetries of RNCQM, as a direct consequence, the new generalized excited relativistic energy states $E_{\mathrm{r}-\mathrm{mc}}^{\mathrm{mcy}}\left(n, \delta, \eta, b, A, V_{0}, V_{0}^{\prime}, j, l, s, m\right) \quad$ produced with DVSMCY-Ps, are the sum of the square roots of the shift energy $\left[\Delta E_{\text {mcy }}^{\text {tot }}\left(n, \delta, \eta, b, A, V_{0}, V_{0}^{\prime}, \Theta, \sigma, \chi, j, l, s, m\right)\right]^{1 / 2}$ and the usual relativistic energy $E_{n l}$ due to the effect of the equal vector scalar Manning-Rosen plus a class of Yukawa potentials in RQM symmetries in Eq. (2.8), as follows:

$$
\begin{aligned}
& E_{\mathrm{r}-\mathrm{nc}}^{\mathrm{mcy}}\left(n, \delta, \eta, b, A, V_{0}, V_{0}^{\prime}, \Theta, \sigma, \chi, j, l, s, m\right) \\
& =E_{n l}+\left[\langle\Xi\rangle_{(n l m)}^{\mathrm{RMCY}}\left(n, \delta, \eta, b, A, V_{0}, V_{0}^{\prime}\right)(\Theta k(j, l, s)+\sigma B m+m \chi \Omega m)\right]^{1 / 2} .
\end{aligned}
$$

For the ground state and first excited state, the above equation can be reduced to the following form:

$$
\begin{aligned}
& E_{\mathrm{r}-\mathrm{nc}}^{\mathrm{mcy}}\left(n=0, \delta, \eta, b, A, V_{0}, V_{0}^{\prime}, \Theta, \sigma, \chi, j, l, s, m\right) \\
& =E_{0 l}+\left[\langle\Xi\rangle_{(n l m)}^{\mathrm{RMCY}}\left(n=0, \delta, \eta, b, A, V_{0}, V_{0}^{\prime}\right)(\Theta k(j, l, s)+\sigma B m+m \chi \Omega m)\right]^{1 / 2}
\end{aligned}
$$

and

$$
\begin{aligned}
& E_{\mathrm{r}-\mathrm{nc}}^{\mathrm{mcy}}\left(n=1, \delta, \eta, b, A, V_{0}, V_{0}^{\prime}, \Theta, \sigma, \chi, j, l, s, m\right) \\
& =E_{1 l}+\left[\langle\Xi\rangle_{(n l m)}^{\mathrm{RMCY}}\left(n=1, \delta, \eta, b, A, V_{0}, V_{0}^{\prime}\right)(\Theta k(j, l, s)+\sigma B m+m \chi \Omega m)\right]^{1 / 2} .
\end{aligned}
$$

Equation (4.4) describe the relativistic energy of some diatomic molecules such as $\mathrm{HCl}, \mathrm{CH}$, $\mathrm{LiH}$, and $\mathrm{CO}$ under the deformed equal vector scalar Manning-Rosen plus a class of Yukawa potentials in RNCQM symmetries. 


\section{A. Relativistic particular cases under MESVEMHPs}

After examining the bound state solutions of any $l$-state deformed Klein-Gordon equation with the deformed equal vector scalar Manning-Rosen plus a class of Yukawa potentials in RNCQM symmetries, our task is now to discuss some particular cases below. By adjusting potential parameters for each case, some familiar potentials, which are useful for other physical systems, can be obtained.

(1) Upon setting $V_{0}$ and $V_{0}^{\prime}$ to zero, the potential in
Eq. (2.1) turns into the deformed equal vector scalar Manning-Rosen [16] in RQM symmetries, as follows :

$$
V_{\mathrm{mp}}(r)=\frac{1}{2 M b^{2}}\left[\frac{\eta(\eta-1) e^{-2 r / b}}{\left(1-e^{-r / b}\right)^{2}}-\frac{A e^{-r / b}}{1-e^{-r / b}}\right]
$$

The perturbed effective potential $V_{\text {pert }}^{\text {mcy }}(r)$ in Eq. (3.18) turns to the perturbed effective potential $V_{\text {pert }}^{\mathrm{mp}}(r)$ in the symmetries of RNCQM as follows:

$$
\begin{aligned}
& V_{\text {pert }}^{\mathrm{mp}}(r)=\frac{16 \delta^{4} l(l+1) s^{2}}{(1-s)^{4}} \mathbf{L} \Theta-2 \delta\left(E_{n l}^{\mathrm{mp}}+M\right) \\
& \times\left(-\frac{8 \delta^{3}}{M} \eta(\eta-1) \frac{s^{5 / 2}}{(1-s)^{3}}-\frac{8 \delta^{3}}{M} \eta(\eta-1) \frac{s^{7 / 2}}{(1-s)^{4}}+\frac{8 \delta^{3} A}{M} \frac{s^{3 / 2}}{(1-s)^{2}}\right) \mathbf{L} \Theta+O\left(\Theta^{2}\right) .
\end{aligned}
$$

In this case, the additive energy shift $\Delta E_{\mathrm{mp}}^{\mathrm{tot}}(n, \delta, \eta, b, A, j, l, s, m)$ under the modified influence of the equally mixed Manning-Rosen potentials in RNCQM symmetries is determined from the following formula:

$$
\Delta E_{\mathrm{mp}}^{\mathrm{tot}}(n, \delta, \eta, b, A, \Theta, \sigma, \chi, j, l, s, m)=\langle\Xi\rangle_{(n l m)}^{\mathrm{RMP}}(n, \delta, \eta, b, A)(\Theta k(j, l, s)+\sigma B+m \chi \Omega m) .
$$

Thus, the corresponding global expectation value $\langle\Xi\rangle_{(n l m)}^{\mathrm{RMP}}(n, \delta, \eta, b, A)$ is determined from the following expression:

$$
\begin{aligned}
& \langle\Xi\rangle_{(n l m)}^{\mathrm{RMP}}(n, \delta, \eta, b, A)=16 \delta^{4} l(l+1)\left\langle\frac{s^{2}}{(1-s)^{4}}\right\rangle_{(n l m)}-2 \delta\left(E_{n l}^{\mathrm{mp}}+M\right) \\
& \times\left(-\frac{8 \delta^{3}}{M} \eta(\eta-1)\left(\left\langle\frac{s^{5 / 2}}{(1-s)^{3}}\right\rangle_{(\mathrm{nlm})}+\left\langle\frac{s^{7 / 2}}{(1-s)^{4}}\right\rangle_{(n l m)}\right)+\frac{8 \delta^{3} A}{M}\left\langle\frac{s^{3 / 2}}{(1-s)^{2}}\right\rangle_{(\mathrm{nlm})}\right) .
\end{aligned}
$$

The new relativistic energy in Eq. (4.2) reduces to the new energy $E_{\mathrm{r}-\mathrm{nc}}^{\mathrm{mp}}\left(n, \delta, \eta, b, A, V_{0}, V_{0}^{\prime}, \Theta, \sigma, \chi, j, l, s, m\right)$ under the modified equal scalar and vector new Manning-Rosen potential in RNCQM, as follows:

$$
E_{\mathrm{r}-\mathrm{nc}}^{\mathrm{mp}}\left(n, \delta, \eta, b, A, V_{0}, V_{0}^{\prime}, \Theta, \sigma, \chi, j, l, s, m\right)=E_{n l}^{\mathrm{mp}}+\left[\begin{array}{c}
\langle\Xi\rangle_{(n l m)}^{\mathrm{RMP}}(n, \delta, \eta, b, A) \\
(\Theta k(j, l, s)+\sigma B m+m \chi \Omega m)
\end{array}\right]^{1 / 2} .
$$

Making the corresponding parameter replacements in Eq. (2.8), we obtain the energy equation for the ManningRosen potentials in the Klein-Gordon theory with equally mixed potentials, in RQM symmetries as :

$$
M^{2}-E_{n l}^{\mathrm{mp} 2}=\delta^{2}\left[\frac{\beta_{n l}^{\mathrm{mp} 2}-l(l+1)-1 / 2-n(n+1)}{n+1 / 2+\sqrt{1 / 4+\alpha_{n l}^{2}+l(l+1)}}-\frac{(2 n+1) \sqrt{1 / 4+\alpha_{n l}^{\mathrm{mp} 2}+l(l+1)}}{n+1 / 2+\sqrt{1 / 4+\alpha_{n l}^{2}+l(l+1)}}\right]^{2}
$$

where $\alpha_{n l}^{\mathrm{mp} 2}=\left(E_{n l}^{\mathrm{mp}}+M\right) \frac{\delta \eta(\eta-1)}{M}$ and $\beta_{n l}^{\mathrm{mp} 2}=$ $\left(E_{n l}^{\mathrm{mp}}+M\right) \frac{\delta A}{M}$. It should be noted that the result recorded in Eq. (4.9) is consistent with the results of our research in Ref. [88].

(2) Upon setting $\eta$ and $A$ to zero, the potential in Eq. (2.1) turns into the deformed equal vector scalar class of Yukawa potentials in RQM symmetries, as follows:

$$
V_{\text {cyp }}(r)=-\frac{V_{0} e^{-\delta r}}{r}-\frac{V_{0}^{\prime} e^{-2 \delta r}}{r^{2}}
$$

The perturbed effective potential $V_{\text {pert }}^{\text {mcy }}(r)$ in Eq. (3.18) turns into the perturbed effective potential $V_{\text {pert }}^{\text {cyp }}(r)$ in the symmetries of RNCQM as follows: 


\section{A. MAIRECHE}

$$
V_{\text {pert }}^{\text {mcy }}(r)=\frac{16 \delta^{4} l(l+1) s^{2}}{(1-s)^{4}} \mathbf{L} \Theta-2 \delta\left(E_{n l}^{\text {cyp }}+M\right)\left(\frac{8 \delta^{3} V_{0} s^{5 / 2}}{(1-s)^{3}}+\frac{2 V_{0} \delta^{2} s^{3 / 2}}{(1-s)^{2}}+\frac{4 V_{0} \delta^{2} s^{2}}{(1-s)^{3}}+\frac{16 V_{0}^{\prime} \delta^{3} s^{3}}{(1-s)^{4}}\right) \mathbf{L} \Theta .
$$

In this case, the additive energy shift $\Delta E_{\mathrm{cyp}}^{\mathrm{tot}}\left(n, \delta, V_{0}, V_{0}^{\prime}, j, l, s, m\right)$ under the influence of the modified equally mixed class of Yukawa potentials in RNCQM symmetries is determined from the following formula:

$$
\Delta E_{\mathrm{mcy}}^{\mathrm{tot}}\left(n, \delta, V_{0}, V_{0}^{\prime}, \Theta, \sigma, \chi, j, l, s, m\right)=\langle\Xi\rangle_{(n l m)}^{\mathrm{RCYP}}(n, \delta, \eta, b, A)(\Theta k(j, l, s)+\sigma B+m \chi \Omega m) .
$$

Thus, the corresponding global expectation value $\langle\Xi\rangle_{(n l m)}^{\mathrm{RMP}}(n, \delta, \eta, b, A)$ is determined from the following expression:

$$
\begin{aligned}
& \langle\Xi\rangle_{(n l m)}^{\mathrm{RCYP}}\left(n, \delta, V_{0}, V_{0}^{\prime}\right)=16 \delta^{4} l(l+1)\left\langle\frac{s^{2}}{(1-s)^{4}}\right\rangle_{(n l m)}-4 \delta^{3}\left(E_{n l}^{\mathrm{cyp}}+M\right) \\
& \times\left\langle 4 \delta V_{0}\left\langle\frac{s^{5 / 2}}{(1-s)^{3}}\right\rangle_{(n l m)}+V_{0}\left\langle\frac{s^{3 / 2}}{(1-s)^{2}}\right\rangle_{(n l m)}+2 V_{0}\left\langle\frac{s^{2}}{(1-s)^{3}}\right\rangle_{(n l m)}+8 V_{0}^{\prime} \delta\left\langle\frac{s^{3}}{(1-s)^{4}}\right\rangle_{(n l m)}\right)
\end{aligned}
$$

The new relativistic energy in Eq. (4.2) is reduced to the new energy $E_{\mathrm{r} \text {-nc }}^{\mathrm{mp}}\left(n, \delta, V_{0}, V_{0}^{\prime}, \Theta, \sigma, \chi, j, l, s, m\right)$ under the modified equal scalar and vector new Manning-Rosen potential in RNCQM, as follows:

$$
E_{\mathrm{r}-\mathrm{nc}}^{\mathrm{cyp}}\left(n, \delta, V_{0}, V_{0}^{\prime}, \Theta, \sigma, \chi, \Theta, \sigma, \chi, j, l, s, m\right)=E_{n l}^{\mathrm{cyp}}+\left[\begin{array}{c}
\langle\Xi\rangle_{(n l m)}^{\mathrm{RCYP}}\left(n, \delta, V_{0}, V_{0}^{\prime}\right) \\
(\Theta k(j, l, s)+\sigma B m+m \chi \Omega m)
\end{array}\right]^{1 / 2} .
$$

Making the corresponding parameter replacements in Eq. (2.8), we obtain the energy equation for the class of Yukawa potentials in the Klein-Gordon theory with equally mixed potentials, in RQM symmetries as:

$$
M^{2}-E_{n l}^{\mathrm{cyp} 2}=\delta^{2}\left[\begin{array}{c}
\frac{\left(E_{n l}^{\mathrm{cyp}}+M\right) V_{0}-l(l+1)-1 / 2}{n+1 / 2+\sqrt{1 / 4+l(l+1)}} \\
\frac{-n(n+1)-(2 n+1) \sqrt{1 / 4+l(l+1)}}{n+1 / 2+\sqrt{1 / 4+l(l+1)}}
\end{array}\right]^{2}
$$

\section{NONRELATIVISTIC SPECTRUM UNDER DVSMCY-Ps}

In this section, we want to derive the nonrelativistic spectrum, which is produced with the effect of the deformed Manning-Rosen plus a class of Yukawa potentials for the diatomic molecules such as $\mathrm{HCl}, \mathrm{CH}, \mathrm{LiH}$, and $\mathrm{CO}$. From Eq. (1.1), we can write the modified Manning-Rosen plus a class of Yukawa potentials in the nonrelativistic noncommutative three-dimensional real space NRNCQM symmetries as follows:

$$
V_{\mathrm{nc}}^{\mathrm{mcy}}(r)=\frac{1}{2 M b^{2}}\left[\frac{\eta(\eta-1) e^{-2 r / b}}{\left(1-e^{-r / b}\right)^{2}}-\frac{A e^{-r / b}}{1-e^{-r / b}}\right]-\frac{V_{0} e^{-\delta r}}{r}-\frac{V_{0}^{\prime} e^{-2 \delta r}}{r^{2}}+V_{\mathrm{nr}-\text { pert }}^{\mathrm{mcy}}(r),
$$

where $V_{\text {pert }}^{\text {mcy }}(r)$ is the perturbative potential in nonrelativistic noncommutative three-dimensional real space NRNCQM symmetries:

$$
V_{\mathrm{nr}-\text { pert }}^{\text {mcy }}(r)=\frac{l(l+1)}{r^{4}} \mathbf{L} \Theta-\frac{\partial V_{\mathrm{nc}}^{\mathrm{mcy}}(r)}{\partial r} \frac{\mathbf{L} \Theta}{2 r}+O\left(\Theta^{2}\right) .
$$

The first term is due to the centrifugal term $\frac{l(l+1)}{r_{n c}^{2}}$ in NRNCQM [Eq. (3.7)], which equals the usual centrifugal term $\frac{l(l+1)}{r^{2}}$ plus the perturbative centrifugal term $\frac{l(l+1)}{r^{4}} \mathbf{L} \Theta$, while the second term in Eq. (5.2) is produced with the effect of the deformed Manning-Rosen plus a class of Yukawa potentials. We have applied the approximations type suggested by Greene and Aldrich and Dong et al. to a short-range potential that is an excellent approximation to the centrifugal term for the Manning-Rosen plus a class of Yukawa potentials, and we calculate $\frac{\partial V_{\mathrm{nc}}^{\mathrm{mcy}}(r)}{\partial r}[\mathrm{see}$ Eq. (3.18)]. 
Now, substituting Eq. (3.16) into Eq. (5.2) we apply the approximation to Eq. (3.12), and we get the perturbative potential in NRNCQM symmetries as follows:

$$
\begin{aligned}
V_{\text {nr-pert }}^{\text {mcy }}(r) & =\frac{16 \delta^{4} l(l+1) s^{2}}{(1-s)^{4}} \mathbf{L} \Theta \\
& -\delta\left(\lambda_{1} \frac{s^{5 / 2}}{(1-s)^{3}}+\lambda_{2} \frac{s^{7 / 2}}{(1-s)^{4}}+\lambda_{3} \frac{s^{3 / 2}}{(1-s)^{2}}+\lambda_{4} \frac{s^{2}}{(1-s)^{3}}+\lambda_{5} \frac{s^{3}}{(1-s)^{4}}\right) \mathbf{L} \Theta+O\left(\Theta^{2}\right) .
\end{aligned}
$$

Thus, we need to know the expectation values of $\frac{s^{2}}{(1-s)^{4}}, \frac{s^{5 / 2}}{(1-s)^{3}}, \frac{s^{7 / 2}}{(1-s)^{4}}, \frac{s^{3 / 2}}{(1-s)^{2}}, \frac{s^{2}}{(1-s)^{3}}$, and $\frac{s^{3}}{(1-s)^{4}}$ to find the nonrelativistic energy corrections produced with the perturbative potential $V_{\mathrm{nr} \text {-pert }}^{\text {mcy }}(r)$. By using the expectations values obtained in Eqs. (3.32)-(3.37) for the $n^{\text {th }}$ excited state we get the corresponding global expectation values $\langle\Xi\rangle_{(n l m)}^{\mathrm{NRMCY}}\left(n, \delta, \eta, b, A, V_{0}, V_{0}^{\prime}\right)$ as follows:

$$
\begin{aligned}
& \langle\Xi\rangle_{(n l m)}^{\mathrm{NRMCY}}\left(n, \delta, \eta, b, A, V_{0}, V_{0}^{\prime}\right)=16 \delta^{4} l(l+1)\left\langle\frac{s^{2}}{(1-s)^{4}}\right\rangle_{(n l m)} \\
& -\delta\left(\lambda_{1}\left\langle\frac{s^{5 / 2}}{(1-s)^{3}}\right\rangle_{(n l m)}+\lambda_{2}\left\langle\frac{s^{7 / 2}}{(1-s)^{4}}\right\rangle_{(n l m)}\right) \\
& -\delta\left(+\lambda_{3}\left\langle\frac{s^{3 / 2}}{(1-s)^{2}}\right\rangle_{(n l m)}+\lambda_{4}\left\langle\frac{s^{2}}{(1-s)^{3}}\right\rangle_{(n l m)}+\lambda_{5}\left\langle\frac{s^{3}}{(1-s)^{4}}\right\rangle_{(\mathrm{nlm})}\right) .
\end{aligned}
$$

By following the same physical methodology that we used in our previous relativistic study, we obtain the energy corrections $\Delta E_{\mathrm{mcy}}^{\mathrm{nr}}\left(n, \delta, \eta, b, A, V_{0}, V_{0}^{\prime}, \Theta, \sigma, \chi, j, l, s, m\right)$ for the $n^{\text {th }}$ excited state due to the spin-orbit complying, the modified Zeeman effect, and the nonrelativistic perturbed Fermi gas potential, which is induced by the influence of DVSMCY-Ps in NRNCQM symmetries, as follows:

$$
\begin{aligned}
& \Delta E_{\mathrm{mcy}}^{\mathrm{nr}}\left(n, \delta, \eta, b, A, V_{0}, V_{0}^{\prime}, \Theta, \sigma, \chi, j, l, s, m\right) \\
& =\langle\Xi\rangle_{(n l m)}^{\mathrm{NRMCY}}\left(n, \delta, \eta, b, A V_{0}, V_{0}^{\prime}\right)(\Theta k(j, l, s)+\sigma B+m \chi \Omega m) .
\end{aligned}
$$

As a direct consequence, the new nonrelativistic energy $E_{\mathrm{nr}-\mathrm{mc}}^{\mathrm{mcy}}\left(n, \delta, \eta, b, A, V_{0}, V_{0}^{\prime}, \Theta, \sigma, \chi, j, l, s, m\right)$ produced with DVSMCY-Ps, in the symmetries of NRNCQM, corresponding the generalized excited states; the sum of the energy corrections $\Delta E_{\mathrm{mcy}}^{\mathrm{nr}}\left(n, \delta, \eta, b, A, V_{0}, V_{0}^{\prime}, \Theta, \sigma, \chi, j, l, s, m\right)$ plus the nonrelativistic energy $E_{n l}^{\mathrm{nr}}$ produced with the main part of potential in Eq. (1.1) in NRQM are as follows:

$$
\begin{aligned}
& E_{\mathrm{r}-\mathrm{nc}}^{\mathrm{mcy}}\left(n, \delta, \eta, b, A, V_{0}, V_{0}^{\prime}, \Theta, \sigma, \chi, j, l, s, m\right) \\
& =E_{n l}^{\mathrm{nr}}+\langle\Xi\rangle_{(n l m)}^{\mathrm{NRMCY}}\left(n, \delta, \eta, b, A V_{0}, V_{0}^{\prime}\right)(\Theta k(j, l, s)+\sigma B+m \chi \Omega m) .
\end{aligned}
$$

The nonrelativistic energy $E_{n l}^{\mathrm{nr}}$ due to the effect of the Manning-Rosen plus a class of Yukawa potentials in NRQM is determined directly from the study of B. I. Ita et al. [89] given by:

$$
E_{n l}^{\mathrm{nr}}=-\frac{\alpha^{2}}{2}\left(\frac{2 \mu V_{0}}{\alpha}-(l+1 / 2)^{2}\left[2(l+1 / 2)^{2}-\frac{2 \mu C}{\alpha^{2}}+(n+1 / 2)^{2}-\frac{2 \mu V_{0}}{\alpha}-\frac{2 \mu V_{1}}{\alpha}+(2 n+1) \tau\right]^{2}\right)
$$




\section{A. MAIRECHE}

With $\tau=\sqrt{(l+1 / 2)^{2}-\frac{2 \mu C}{\alpha^{2}}-\frac{2 \mu V_{0}}{\alpha}-\frac{2 \mu V_{1}}{\alpha}}, \alpha \rightarrow \delta$, $V_{0} \rightarrow 0, D \rightarrow-\eta(\eta-1), V_{2} \rightarrow V_{0}^{\prime}, C \rightarrow A$ and $V_{1} \rightarrow V_{0}$ to match the notations used in the two references are identical.

Now, considering composite systems such as molecules made of $N=2$ particles of masses $m_{n}(n=1,2)$ within the frame of noncommutative algebra, it is worth taking into account features of descriptions of the systems in the space. In NRQM symmetries, it was obtained that composite systems with different masses are described with different noncommutative parameters [90-93]:

$$
\left[\hat{x}_{\mu}^{\mathrm{S}}, \hat{x}_{\nu}^{\mathrm{S}}\right]=\left[\hat{x}_{\mu}^{\mathrm{H} *}, \hat{x}_{\nu}^{\mathrm{H}}\right]=\left[\hat{x}_{\mu}^{\mathrm{I}}, \hat{x}_{\nu}^{\mathrm{I}}\right]=i \theta_{\mu \nu}^{c},
$$

where the noncommutativity parameter $\theta_{\mu \nu}^{c}$ is given by:

$$
\theta_{\mu \nu}^{c}=\sum_{n=1}^{2} \mu_{n}^{2} \theta_{\mu \nu}^{(n)}
$$

with $\mu_{n}=\frac{m_{n}}{\sum_{n} m_{n}}$, the indices $(n=1,2)$ label the particles, and $\theta_{\mu \nu}^{(n)}$ is the parameter of noncommutativity, corresponding to the particle of mass $m_{n}$. Note that in the case of a system of two particles with the same mass $m_{1}=m_{2}$ such as the homogeneous $\left(\mathrm{N}_{2}\right.$ and $\left.\mathrm{I}_{2}\right)$ diatomic molecules, the parameter $\theta_{\mu \nu}^{(n)}=\theta_{\mu \nu}$. Thus, the two parameters $\Theta$ and $\sigma$ which appears in Eq. (5.8) are changed to the new form:

$$
\left\{\begin{array}{c}
\Theta^{c 2}=\left(\sum_{n=1}^{2} \mu_{n}^{2} \Theta_{12}^{(n)}\right)^{2}+\left(\sum_{n=1}^{2} \mu_{n}^{2} \Theta_{23}^{(n)}\right)^{2}+\left(\sum_{n=1}^{2} \mu_{n}^{2} \Theta_{13}^{(n)}\right)^{2} \\
\sigma^{c 2}=\left(\sum_{n=1}^{2} \mu_{n}^{2} \sigma_{12}^{(n)}\right)^{2}+\left(\sum_{n=1}^{2} \mu_{n}^{2} \sigma_{23}^{(n)}\right)^{2}+\left(\sum_{n=1}^{2} \mu_{n}^{2} \sigma_{13}^{(n)}\right)^{2} \\
\chi^{c 2}=\left(\sum_{n=1}^{2} \mu \chi_{12}^{(n)}\right)^{2}+\left(\sum_{n=1}^{2} \mu \chi_{23}^{(n)}\right)^{2}+\left(\sum_{n=1}^{2} \mu \chi_{13}^{(n)}\right)^{2}
\end{array}\right.
$$

As is mentioned above, in the case of a system of two particles with the same mass $m_{1}=m_{2}$ such as the homogeneous $\left(\mathrm{N}_{2}\right.$ and $\left.\mathrm{I}_{2}\right)$ diatomic molecules $\Theta_{\mu \nu}^{(n)}=\Theta_{\mu \nu}$ and $\sigma_{\mu \nu}^{(n)}=\sigma_{\mu \nu}$. Finally, we can generalize the nonrelativistic global energy $E_{\mathrm{r}-\mathrm{nc}}^{\mathrm{mcy}}\left(n, \delta, \eta, b, A, V_{0}, V_{0}^{\prime}, j, l, s, m\right)$ under the modified Morse potential considering that composite systems with different masses are described with different noncommutative parameters for the diatomic $(\mathrm{HCl}, \mathrm{CH}$, $\mathrm{LiH}$, and $\mathrm{CO}$ ) as:

$$
\begin{aligned}
& E_{\mathrm{r}-\mathrm{nc}}^{\mathrm{mcy}}\left(n, \delta, \eta, b, A, V_{0}, V_{0}^{\prime}, j, l, s, m\right) \\
& =E_{n l}^{\mathrm{nr}}+\langle\Xi\rangle_{(n l m)}^{\mathrm{NRMCY}}\left(n, \delta, \eta, b, A V_{0}, V_{0}^{\prime}\right) \\
& \times\left(\Theta^{c} k(j, l, s)+\sigma^{c} B+m \chi^{c} \Omega m\right) .
\end{aligned}
$$

The KGE is the most well-known relativistic wave equation describing spin-zero particles, but its extension in RNCQM symmetries, DKGE, under the modified Manning-Rosen plus a class of Yukawa potentials model has a physical behavior similar to the Duffin-Kemmer equation for meson with spin-1; it can describe a dynamic state of a particle with spin one in the symmetries of relativistic noncommutative quantum mechanics. This is one of the most important new results of this research. It is worth mentioning that for the two simultaneous limits $(\Theta, \sigma, \chi)$ and $\left(\Theta^{c}, \sigma^{c}, \chi^{c}\right) \rightarrow(0,0)$, we recover the results of the in Refs. [32, 90].

\section{SUMMARY AND CONCLUSION}

In this work, we have found approximate bound state solutions of DRKGE and DNRSE using the tool of Bopp's shift and standard perturbation theory methods of the deformed equal vector scalar Manning-Rosen plus a class of Yukawa potentials in both relativistic and nonrelativistic regimes, which correspond to high and low energy physics. We have employed the improved approximation scheme to deal with the centrifugal term to obtain the new relativistic bound state solutions $\quad E_{\mathrm{r}-\mathrm{nc}}^{\mathrm{mcy}}\left(n, \delta, \eta, b, A, V_{0}, V_{0}^{\prime}, \Theta, \sigma, \chi, j, l, s, m\right)$ corresponding to the generalized excited states that appear as a sum of the total shift energy $\Delta E_{\text {mcy }}^{\text {tot }}\left(n, \delta, \eta, b, A, V_{0}, V_{0}^{\prime}, \Theta, \sigma, \chi, j, l, s, m\right)$ and the relativistic energy $E_{n l}$ of the equal vector scalar Manning-Rosen plus a class of Yukawa potentials. Furthermore, we have obtained the new nonrelativistic global energy of some diatomic molecules, such as $\mathrm{N}_{2}$, $\mathrm{I}_{2}, \mathrm{HCl}, \mathrm{CH}, \mathrm{LiH}$, and CO, in NRNCQM symmetries as a sum of the nonrelativistic energy and the perturbative corrections. The total shift energy and the perturbative corrections appear as a function of the discreet atomic quantum numbers $(n, j, l, s, m)$, the potential parameters $\left(\delta, \eta, b, A, V_{0}, V_{0}^{\prime}\right)$ in addition to three noncommutativity parameters $(\Theta, \sigma, \chi)$. This behavior is similar to the perturbed both modified Zeeman effect and modified perturbed spin-orbit coupling in which an external magnetic field is applied to the system and the spinorbit couplings which are generated with the effect of 
the perturbed effective potential in the symmetries of RNCQM and NRNCQM. Furthermore, we can conclude that the DKGE under the DVSMCY-Ps model becomes similar to the Duffin-Kemmer equation for meson with spin-1; it can describe a dynamic state of a particle with spin one in the symmetries of RNCQM. Furthermore, we have applied our results to composite systems such as molecules made of $N=2$ particles of masses $m_{n}(n=1,2)$. It is worth mentioning that, for all cases, when to make the two simultaneous limits $(\Theta, \sigma, \chi)$ and
$\left(\Theta^{c}, \sigma^{c}, \chi^{c}\right) \rightarrow(0,0,0)$, the ordinary physical quantities are recovered. Furthermore, our research findings could also be applied in atomic physics, vibrational and rotational spectroscopy, mass spectra, nuclear physics, and other applications. Finally, given the effectiveness of the methods that we followed in achieving our goal in this research, we advise researchers to apply the same methods to delve more deeply, both into the relativistic and nonrelativistic regimes for others potentials.
[1] A. F. Nikiforov, V. B. Uvarov, Special Functions of Mathematical Physics (Birkhäuser, Basel, 1988).

[2] B. I. Ita, et al., Sri Lankan J. Phys. 19, 37 (2018); https : //doi.org/10.4038/sljp.v19i1.8050.

[3] H. Louis et al., Eur. Phys. J. Plus 134, 315 (2019); http $\mathrm{s}: / /$ doi .org/10.1140/epjp/i2019-12835-3.

[4] M. X. Shao et al., Int. J. Theor. Phys. 48, 36 (2009); https://doi.org/10.1007/s10773-008-9779-x.

[5] C. L. Pekeris, Phys. Rev. 45, 98 (1934); https://doi. org/10.1103/physrev.45.98.

[6] R. L. Greene, C. Aldrich, Phys. Rev. A 14, 2363 (1976); https://doi.org/10.1103/physreva.14.2363

[7] B. H. Yazarloo et al., Eur. Phys. J. Plus 127, 51 (2012); https://doi.org/10.1140/epjp/i2012-12051-9.

[8] S. H. Dong et al., Int. J .Mod. Phys. E 16, 189 (2007); https://doi.org/10.1142/S0218301307005661.

[9] M. F. Manning et al., Phys. Rev. 44, 951 (1933); https: //doi.org/10.1103/physrev.44.951.

[10] M. F. Manning, N. Rosen, Phys. Rev. 44, 953 (1933); https://doi.org/10.1103/physrev.44.951.

[11] M. F. Manning, J. Chem. Phys. 3, 136 (1935); https: //doi.org/10.1063/1.1749619.

[12] M. F. Manning, Phys. Rev. 48, 161 (1935); https://do i.org/10.1103/physrev. 48.161.

[13] B. J. Falaye et al., Can. J. Phys. 91, 98 (2013); https: //doi.org/10.1139/cjp-2012-0330.

[14] S. H. Dong, J. García-Ravelo, Phys. Scr. 75, 307 (2007); https://doi.org/10.1088/0031-8949/75/3/013.

[15] S. M. Ikhdair, Phys. Scr. 83, 015010 (2011); https://do i .org/10.1088/0031-8949/83/01/015010.

[16] N. Hatami, M. R. Setare, Indian J. Phys. 91, 1229 (2017); https://doi.org/10.1007/s12648-017-10204.

[17] G. F. Wei et al., Cent. Eur. J. Phys. 7, 175 (2009); https : //doi.org/10.2478/s11534-008-0143-9.

[18] G. F. Wei, S. H. Dong, Phys. Lett. A 373, 49 (2008); https://doi.org/10.1016/j.physleta.2008.10.064.

[19] M. K. Bahar, F. Yasuk, Few-Body Syst. 53, 515 (2012); https://doi.org/10.1007/s00601-012-0461-8.

[20] H. Yukawa, Proc. Phys. Math. Soc. Jpn. 17, 48 (1935).

[21] E. R. Vrscay, Phys. Rev. A 33, 1433 (1986); https://do i.org/10.1103/physreva.33.1433.

[22] J. M. Ugalde et al., Phys. Rev. A 56, 1642 (1997); https: //doi.org/10.1103/physreva.56.1642.

[23] J. P. Gazeau, A. Maquet, Phys. Rev. A 20, 727 (1979); https://doi.org/10.1103/PhysRevA.20.727.

[24] H. Totsuji, J. Phys. Soc. Jpn. 31, 584 (1971); https: //doi.org/10.1143/jpsj.31.584.

[25] C. B. Smith, Phys. Rev. A 134, 1235 (1964) https:// doi .org/10.1103/PhysRev .134.A1235.

[26] G. M. Harris, Phys. Rev. 125, 1131 (1962); https://do i.org/10.1103/PhysRev.125.1131.

[27] J. McEnnan, L. Kissel, R. H. Pratt, Phys. Rev. A 13, 532 (1976); https://doi.org/10.1103/physreva.13.532.

[28] C. H. Mehta, S. H. Patil, Phys. Rev. A 17, 34 (1978); https://doi.org/10.1103/physreva.17.34.

[29] R. Dutt, Y. P. Varshni, Z. Phys. Atom. Nucl. 313, 143 (1983).https : //doi .org/10.1007/bf01417219.

[30] T. O. Magu et al., J. Niger. Assoc. Math. Phys. 42, 1 (2017).

[31] H. Louis et al., Phys. Sci. Int. J. IJCPS 7, 33 (2018); https://doi .org/10.9734/PSIJ/2017/34330.

[32] A. I. Ahmadov et al., Phys. Lett. A 384, 126372 (2020); https://doi.org/10.1016/j.physleta.2020.126372.

[33] S. Capozziello et al., Int. J. Theor. Phys. 39, 15 (2000); https://doi .org/10.1023/A: 1003634814685.

[34] S. Doplicher et al., Phys. Lett. B 331, 39 (1994); https: //doi.org/10.1016/0370-2693 (94)90940-7.

[35] E. Witten, Phys. Today 49, 24 (1996); https://doi.or $\mathrm{g} / 10.1063 / 1.881493$

[36] A. Kempf et al., Phys. Rev. D 52, 1108 (1995); https: //doi.org/10.1103/physrevd.52.1108.

[37] F. Scardigli, Nuovo Cim. B 110, 1029 (1995); https: //doi.org/10.1007/bf02726152.

[38] R. J. Adler, D. I. Santigo, Mod. Phys. Lett. A 14, 1371 (1999); https://doi.org/10.1142/s02177323990 01462.

[39] T. Kanazawa et al., Eur. Phys. J. C 79, art. 95 (2019); https://doi.org/10.1140/epjc/s10052-019-6610-1.

[40] F. Scardigli, Phys. Lett. B 452, 39 (1999); https://do i . org/10.1016/s0370-2693 (99) 00167-7.

[41] P. M. Ho, H. C. Kao, Phys. Rev. Lett. 88, 151602 (2002); https://doi.org/10.1103/physrevlett.88.151602.

[42] P. Gnatenko, Phys. Rev. D 99, 026009 (2019); https: //doi.org/10.1103/physrevd.99.026009.

[43] O. Bertolami et al., Mod. Phys. Lett. A 21, 795 (2006); https://doi .org/10.1142/s0217732306019840.

[44] A. Connes, M. R. Douglas, A. Schwarz, J. High Energy Phys. 1998, 003 https://doi.org/10.1088/1126-670 8/1998/02/003.

[45] A. Maireche, Afr. Rev. Phys. 15, 1 (2020); http://lamp.ictp.it/index.php/aphysrev/arti cle/view/1777/618.

[46] A. Maireche, Int. J. Geom. Meth. Mod. Phys. 17, 2050067 (2020); https ://doi.org/10.1142/S021988782 $050067 \mathrm{X}$.

[47] A. Maireche, J. Nano- Electron. Phys. 9, 03021 (2017); https://doi.org/10.21272/jnep.9(3).03021. 
[48] A. Maireche, Int. Front. Sci. Lett. 11, 29 (2017); https: //doi.org/10.18052/www. scipress. com/IFSL.11.29.

[49] A. Maireche, Few-Body Syst. 61, 30 (2020); https://do i. org/10.1007/s00601-020-01559-z.

[50] A. Maireche, Ukr. J. Phys. 65(11), 987 (2020). https: //doi .org/10.15407/ujpe65.11.987.

[51] A. Maireche, Int. Lett. Chem. Phys. Astron. 76, 1 (2017); https ://doi.org/10.18052/www .scipress .co $\mathrm{m} /$ ILCPA.76.1.

[52] A. Maireche, To Phys. J. 4, 16 (2019); https://purkh. com/index.php/tophy/article/view/521.

[53] J. Gamboa, M. Loewe, J. C. Rojas, Phys. Rev. D 64, 067901 (2001); https://doi.org/10.1103/PhysRevD.6 4.067901 .

[54] E. F. Djemaï, H. Smail, Commun. Theor. Phys. 41, 837 (2004); https ://doi.org/10.1088/0253-6102/41/6 1837.

[55] Y. Yi et al., Chin. Phys. C 34, 543 (2010); https://do i. org/10.1088/1674-1137/34/5/005.

[56] O. Bertolami, P. Leal, Phys. Lett. B 750, 6 (2015).http s://doi.org/10.1016/j.physletb.2015.08.024.

[57] O. Bertolami et al., Phys. Rev. D 72, 025010 (2005); https://doi.org/10.1103/physrevd.72.025010.

[58] J. Zhang, Phys. Lett. B 584, 204 (2004); https://doi. org $/ 10.1016 / j \cdot$ physletb. 2004.01.049.

[59] M. Chaichian et al., Phys. Rev. Lett. 86, 2716 (2001); https://doi.org/10.1103/physrevlett.86.2716.

[60] A. Maireche, J. Nano- Electron. Phys. 11, 04024 (2019); https://doi.org/10.21272/jnep.11(4).04024.

[61] A. Maireche, NanoWorld J. 1(4), 122 (2016); https:// doi.org/10.17756/nwj.2016-016.

[62] M. A. De Andrade, C. Neves, J. Math. Phys. 59, 012105 (2018); https://doi.org/10.1063/1.4986964.

[63] E. M. C. Abreu et al., Int. J. Mod. Phys. A 21, 5359 (2006); https://doi.org/10.1142/s0217751x060 34094.

[64] E. M. C. Abreu et al., Int. J. Mod. Phys. A 27, 1250053 (2012); https://doi.org/10.1142/s0217751x1 2500534 .

[65] L. Mezincescu, preprint arXiv: hep-th/0007046v2 (2000).

[66] J. Wang, K. Li, J. Phys. A 40, 2197 (2007); https://do i. org/10.1088/1751-8113/40/9/021.

[67] K. Li, J. Wang, Eur. Phys. J. C 50, 1007 (2007); https: //doi .org/10.1140/epjc/s10052-007-0256-0.

[68] A. Maireche, Sri Lankan J. Phys. 21, 11 (2020); https: //doi.org/10.4038/sljp.v20i0.

[69] A. Maireche, J. Nano- Electron. Phys. 10, 06015 (2018); https://doi.org/10.21272/jnep.10(6).06015.

[70] P. M. Morse, Phys. Rev. 34, 57 (1929); https://doi.or g/10.1103/physrev.34.57.

[71] P. O. Amadi et al., Rev. Mex. Fís.66, 742 (2020); https:
//doi.org/10.31349/RevMexFis.66.742.

[72] A. Maireche, Mod. Phys. Lett. A 35, 2050015 (2020); https://doi.org/10.1142/s0217732320500157.

[73] A. Maireche, To Phys. J. 3, 186 (2019); https://purkh. com/index.php/tophy/article/view/489.

[74] A. Maireche, Afr. Rev Phys. 15, 19 (2020); http://lamp.ictp.it/index.php/aphysrev/arti cle/view/1779/620.

[75] H. Motavalli, A. R. Akbarieh, Mod. Phys. Lett. A 25, 2523 (2010); https://doi.org/10.1142/s02177323100 33529.

[76] M. Darroodi, H. Mehraban, H. Hassanabadi, Mod. Phys. Lett. A 33, 1850203 (2018); https ://doi .org/10.1142/ s0217732318502036.

[77] A. Saidi, M.B. Sedra, Mod. Phys. Lett. A 35, 2050014 (2019); https://doi.org/10.1142/s021773232 0500145 .

[78] A. Maireche, Sri Lankan J. Phys. 22, 1 (2021); https: //doi.org/10.4038/sljp.v22i1.8079.

[79] A. Maireche, Lat. Am. J. Phys. Educ. 14, 3310 (2020).

[80] L. Gouba et al., Int. J. Mod. Phys. A 31, 1630025 (2016); https://doi.org/10.1142/s0217751x16300258.

[81] F. Bopp, Ann. Inst. Henri Poincaré 15, 81 (1956).

[82] M. Badawi et al., J. Phys. B 5, L157 (1972); https: //doi.org/10.1088/0022-3700/5/8/004.

[83] J. L. Basdevant, Mécanique quantique (Ellipses, 1986) [in French].

[84] S. H. Dong, W. C. Qiang, G. H. Sun, V. B. Bezerra, J. Phys. A 40, 10535 (2007); https://doi.org/10.1088/ $1751-8113 / 40 / 34 / 010$.

[85] Y. Zhang, Phys. Scr. 78, 015006 (2008); https://doi. org/10.1088/0031-8949/78/01/015006S.

[86] K. Bencheikh et al., Phys. Rev. A 89, 063620 (2014); https://doi.org/10.1103/physreva.89.063620.

[87] S. Medjedel, K. Bencheikh, Phys. Lett. A 383, 1915 (2019); https://doi.org/10.1016/j.physleta.2 019.03 .021 .

[88] A. Maireche, Rev. Mex. Fís. 67, 050702 (2021); https: //doi.org/10.31349/RevMexFis.67.050702.

[89] B. I. Ita et al., Bulg. J. Phys. 45, 323 (2018).

[90] K. P. Gnatenko, V. M. Tkachuk, Europhys. Lett. 127, 20008 (2019); https://doi.org/10.1209/0295-5075/12 $7 / 20008$.

[91] K. P. Gnatenko, V. M. Tkachuk, Int. J. Mod. Phys. A 33, 1850037 (2018); https://doi.org/10.1142/s02177 $51 \times 18500379$.

[92] K. P. Gnatenko, Phys. Lett. A 377, 3061 (2013); https: //doi.org/10.1016/j.physleta.2013.09.036.

[93] A. Maireche, J. Phys. Stud. 25, 1002 (2021); https:// doi.org/10.30970/jps.25.1002. 


\title{
НОВІ РОЗВ'ЯЗКИ ДЛЯ ЗВ'ЯЗАНИХ СТАНІВ ДЕФОРМОВАНИХ РІВНЯНЬ КЛЯЙНА-ГОРДОНА ТА ШРЕДИНГЕРА ДЛЯ ДОВІЛЬНОГО L-СТАНУ 3 МОДИФІКОВАНИМИ РІВНИМИ ВЕКТОРНИМ І СКАЛЯРНИМ ПОТЕНЦІАЛАМИ МЕННІНГА-РОЗЕНА ПЛЮС КЛАС ПОТЕНЦІАЛІВ ЮКАВИ В СИМЕТРІЯХ РЕЛЯТИВІСТСЬКОЇ ТА НЕРЕЛЯТИВІСТСЬКОЇ НЕКОМУТАТИВНОї КВАНТОВОї МЕХАНІКИ
}

\author{
Абдельмаджід Майреш \\ Лабораторія фізики та хімї̈ матеріалів, фізичне відділеннл, \\ Факультет природничих наук, Університет Мсіли, Мсіла, Алжир (ВР 239 Шебіліл-Мсіла, Алжир) \\ abdelmadjid.maireche@univ-msila.dz
}

У цій роботі ми використали елегантний інструмент зсуву Боппа та стандартні методи теорії збурень для отримання нового релятивістського й нерелятивістського наближеного розв'язку зв'язаних станів деформованих рівнянь Кляйна-Гордона та Шредингера з використанням модифікованих векторного і скалярного потенціалів Меннінга-Розена плюс клас потенціалів Юкави (скорочено DVSMCY-Ps). Kрім того, ми застосували поліпшене наближення до відцентрового члена для деяких вибраних двоатомних молекул, як-от: $\mathrm{N}_{2}, \mathrm{I}_{2}, \mathrm{HCl}, \mathrm{CH}, \mathrm{LiH}$ та CO, у симетріях розширеної квантової механіки для отримання наближених розв'язків. Енергія релятивістського зсуву $\Delta E_{\mathrm{mcy}}^{\mathrm{tot}}\left(n, \delta, \eta, b, A, V_{0}, V_{0}^{\prime}, \Theta, \sigma, \chi, j, l, s, m\right)$ та пертурбативні нерелятивістські поправки $\Delta E_{\mathrm{mcy}}^{\mathrm{nr}}\left(n, \delta, \eta, b, A, V_{0}, V_{0}^{\prime}, \Theta, \sigma, \chi, j, l, s, m\right)$ вияляються функціями параметрів $\left(\delta, \eta, b, A, V_{0}, V_{0}^{\prime}\right)$, параметрів некомутативності $(\Theta, \sigma, \chi)$, на додаток до атомних квантових чисел $(n, j, l, s, m)$. I в релятивістських, і в нерелятивістських задачах ми показуємо, що поправки на енергію спектра менші за основну в звичайних випадках релятивістської та нерелятивістської квантової механіки. У границі звичайної квантової механіки наші результати для DVSMCY-Рs узгоджуються з тим, що отримано в літературі. У нових симетріях некомутативної квантової механіки неможливо одержати точні аналітичні розв'язки для $l=0$, a $l$ neq0 можна розв'язати лише приблизно. Ми помітили, що деформоване рівняння Кляйна-Гордона з моделлю DVSMCY-Ps має фізичну поведінку, подібну до рівняння Даффіна-Кеммера для мезона зі спіном 1, воно може описувати динамічний стан частинки зі спіном 1 у симетріях релятивістської некомутативної квантової механіки.

Ключові слова: рівняння Кляйна-Гордона, рівняння Шредингера, потенціал Маннінга-Розена, клас потенціалів Юкави, двоатомні молекули, некомутативна геометрія, метод зсуву Боппа та зіркові добутки. 